\title{
BACTERIAL WALL ATTACHMENT IN A FLOW REACTOR*
}

\author{
DON JONES ${ }^{\dagger}$, HRISTO V. KOJOUHAROV $\ddagger$, DUNG LE§, AND HAL SMITH
}

\begin{abstract}
A mathematical model of microbial growth for limiting nutrients in a fully three dimensional flow reactor which accounts for the colonization of the reactor wall surface by the microbes is studied analytically. It can be viewed as a model of the large intestine or of the fouling of a commercial bioreactor or pipe flow. Two steady state regimes are identified, namely, the complete washout of the microbes from the reactor and the successful colonization of both the wall and bulk fluid by the microbes. Only one steady state is stable for any particular set of parameter values. Sharp and explicit conditions are given for the stability of each. The effects of adding an antimicrobial agent to the reactor are examined with and without wall growth.
\end{abstract}

Key words. plug flow, bacterial wall growth, gut, elliptic and parabolic boundary value problem, nonlinear boundary conditions

AMS subject classifications. 92A15, 35K55, 34B25

PII. S0036139901390416

1. Introduction. Recently, a mathematical model of a mixed microbial culture in a flow reactor competing for attachment sites on the reactor wall and based on the work of Freter [18] and Freter, Brickner, and Temme [19] was introduced in [4]. Although the model was motivated by Freter's study of colonization resistance in the mammalian gut (the stability of the natural microflora to invasion by endogenous organisms) it serves as well as a crude model of a biofilm or for the fouling of a commercial bioreactor by wall growth.

In subsequent work [5], the pure culture model was studied. The main result was identifying two possible regimes for the bioreactor which depend only on operating parameters but not on the initial data. These are washout, in which the organism is unable to colonize the reactor, and successful colonization, in which it establishes a steady state characterized by a nonuniform density on the reactor wall as well as in the fluid environment of the reactor. Later work in $[29,7]$ focused on the mixed culture model. In all these previous works the flow reactor was modeled as a thin tube with constant flow velocity so that the model equations took the form of a onespace dimensional system of parabolic or hyperbolic differential equations. However, a three dimensional cylindrical reactor with a realistic steady flow field may not be well approximated using a one-space dimensional model. It is the purpose of this paper to consider the mathematical model in the setting of a three dimensional cylindrical flow reactor.

In the one dimensional model, the reactor wall and the fluid environment reduce to the same interval $0<x<L$ and this means that the interaction terms between the wall attached bacteria and the planktonic bacteria in the fluid are part of the differential equations. However in a three dimensional cylindrical domain, the wall and

* Received by the editors June 5, 2001; accepted for publication (in revised form) December 13, 2001; published electronically June 5, 2002.

http://www.siam.org/journals/siap/62-5/39041.html

${ }^{\dagger}$ Department of Mathematics, Arizona State University, Tempe, AZ 85287-1804.

${ }^{\ddagger}$ Department of Mathematics, University of Texas, Arlington, TX 76019 .

$\S$ Division of Mathematics and Statistics, University of Texas at San Antonio, San Antonio, TX 78249-0664 (dung@lonestar.jpl.utsa.edu).

\Department of Mathematics, Arizona State University, Tempe, AZ 85287-1804 (halsmith@ asu.edu). The research of this author was supported by NSF grant DMS 9700910. 
fluid environment are distinct so the interaction terms are now part of the boundary conditions. As a result, the model equations simplify greatly in passing from the one-space dimensional case to the three dimensional cylindrical geometry, but the boundary conditions become highly nonlinear and strongly coupled. Furthermore, the cylindrical geometry features a nonsmooth domain possessing two edges, complicating the application of standard results for parabolic and elliptic systems.

In this work we show that the basic qualitative conclusions obtained in [5] in the one-space dimensional case carry over with only slight modifications to the fully three dimensional problem with steady Poisseau or constant velocity profile. However, the justification for this requires a substantially more complex argument. Numerical simulations show that steady state solutions can look quite different than their one dimensional counterparts. In addition, we examine the effect of adding an antimicrobial agent to the reactor. We are able to quantify the often observed phenomena that biofilm communities are difficult to control using antimicrobials [11, 12, 31].

The Freter model considered here as a model of a biofilm is far from being a state of the art biofilm model. See [10] for a review of recent modeling in this field. The focus of most recent modeling has been directed toward replicating the remarkable spatial structure of biofilms as seen using the latest imaging techniques $[11,12,10]$. The Freter model, in contrast, ignores completely the spatial structure of the biofilm. However, our reading of the biofilm literature suggests the lack of a relatively simple conceptual model which is amenable to mathematical analysis. We believe the Freter model is this missing piece and this belief has motivated our work on it $[4,5,6,7,29,30]$. It has the useful feature that biofilm formation can be simulated directly, starting from an inoculum of planktonic bacteria, in contrast to most models for which an initial biofilm must be set up. Mathematical tractability of the Freter model is essentially due to the assumption that the biofilm growing on the cylindrical surface is infinitesimally thin (spatial structure of the biofilm is ignored) and consequently that it does not affect the fluid velocity field, which we take to be a steady Poisseau flow. While this assumption is clearly unrealistic (see, e.g., $[12,10]$ ), we believe that the results obtained here can serve as a reference point from which to examine more realistic scenarios. State of the art biofilm models which account for the spatial structure of the biofilm are, with few exceptions [14], too complicated for mathematical analysis $[13,10]$; numerical simulations then provide the only window through which to study these models.

2. The model. A fully three dimensional model incorporating microbial growth on the inner surface of a cylindrical tube $\Omega \equiv\left\{0<x<L, 0 \leq r^{2}=y^{2}+z^{2}<\right.$ $\left.R^{2}\right\}$ under steady flow $v(r)=V_{\max }\left[1-\epsilon(r / R)^{2}\right]$ conditions is formulated following $[18,19,4]$. Here, $\epsilon=0,1$ are of primary interest; $\epsilon=1$ corresponds to Poisseau flow of fluid medium through the reactor. The equations for nutrient density $S=S(x, y, z, t)$, planktonic biomass density $u=u(x, y, z, t)$, and the areal density of wall-attached cells $w=w(x, y, z, t)$ on the radial boundary $(r=R)$ for a single strain are given by (compare with [5])

$$
\begin{aligned}
& S_{t}=d_{x}^{S} S_{x x}+d_{r}^{S}\left[S_{y y}+S_{z z}\right]-v(r) S_{x}-\gamma^{-1} u f_{u}(S), \\
& u_{t}=d_{x}^{u} u_{x x}+d_{r}^{u}\left[u_{y y}+u_{z z}\right]-v(r) u_{x}+u\left(f_{u}(S)-k\right)
\end{aligned}
$$

for $(x, y, z) \in \Omega$. Growth of bacteria on the wall $r=R$ is described by

$$
w_{t}=w\left[f_{w}(S) G(W)-k_{w}-\beta\right]+\alpha u(1-W) .
$$


The specific growth rate of bacteria in the fluid $f_{u}(S)$ and on the wall $f_{w}(S)$ are further described below. Constants $k$ and $k_{w}$ represent cell death rates in fluid and wall environments, respectively, $\beta$ is the rate of sloughing of wall-attached bacteria into the fluid, and $\alpha$ is the rate coefficient of adhesion to the wall of the reactor. A model assumption is that there is a maximum attainable areal density of wall-attached bacteria $w_{\infty}$ and that

$$
W=\frac{w}{w_{\infty}}
$$

is the occupation fraction. Danckwerts' boundary conditions describe the interface conditions between the up-stream and down-stream flow and the reactor. These are as follows (see [3]): at $x=0$,

$$
\begin{aligned}
v(r) S^{0} & =-d_{x}^{S} S_{x}+v(r) S, \\
0 & =-d_{x}^{u} u_{x}+v(r) u,
\end{aligned}
$$

at $x=L$,

$$
S_{x}=u_{x}=0
$$

These conditions reflect the assumption that up-stream flow brings sterile nutrient at concentration $S^{0}$ into the reactor at $x=0$ and flushes out planktonic cells and unused nutrient at $x=L$. The radial boundary conditions at $r=R$ reflect important biological considerations:

$$
\begin{aligned}
& 0=d_{r}^{S} S_{r}+\gamma^{-1} w f_{w}(S), \\
& 0=d_{r}^{u} u_{r}+\alpha u(1-W)-w\left[f_{w}(S)(1-G(W))+\beta\right] .
\end{aligned}
$$

These boundary conditions describe the fluxes of nutrient and biomass between the fluid and wall environment. The first describes the flux of nutrient from the fluid to the wall environment due to consumption by wall-attached bacteria. The first term in the second equation represents the flux of biomass from the fluid to the wall due to passive attraction of planktonic cells to the wall; the second term represents flux in the opposite direction caused by a fraction of the progeny of wall-attached cells being forced into the fluid. These are further described below. In addition, $S, u, w$ satisfy (nonnegative) initial conditions at $t=0$ :

$$
\begin{gathered}
S(x, y, z, 0)=S_{0}(x, y, z), \\
u(x, y, z, 0)=u_{0}(x, y, z), \\
w(x, y, z, 0)=w_{0}(x, y, z) .
\end{gathered}
$$

We assume that $S_{0}, u_{0}, w_{0}$ are continuous.

See $[4,5]$ for a careful description of the modeling for the one dimensional flow reactor. The Freter model is based on the following assumptions. First, there is a maximum attainable biomass density of wall-attached bacteria $w_{\infty}$. Planktonic bacteria are attracted to the wall at a rate proportional to planktonic cell density and the fraction of available colonization sites on the wall, i.e., at rate $\alpha u(1-W)$ (see also [8]). Wall-attached cells are sloughed off into the fluid at a rate proportional to their density. Third, daughter cells of wall-attached bacteria compete for space on the wall. A fraction $G$ of the daughter cells find attachment sites and the fraction 
$1-G$ do not and are forced into the fluid. It is reasonable to assume that $G=G(W)$ is a decreasing function of the occupation fraction $W$ because a more fully saturated wall provides less chance for a daughter cell to find space on it.

It will also be of interest to allow the initial "charging" of the reactor with microbes to take place via the boundary condition at $x=0$ by replacing zero on the left side of (2.4) by $v(r) u^{0}(t)$, where $u^{0}(t) \equiv u^{0}$, a constant, on $0 \leq t \leq t_{0}$ and $u^{0}(t)=0, t \geq t_{0}$.

Although it is usual to nondimensionalize variables, we avoid it so that the biological meaning of key quantities will be transparent. Keeping in mind that $0 \leq w \leq w_{\infty}$, the initial data and solutions $S, u, w$ must satisfy

$$
S \geq 0, \quad u \geq 0, \quad 0 \leq w \leq w_{\infty} .
$$

Hereafter, we refer to these restrictions as the range conditions.

For brevity, we let $L^{S}$ and $L^{u}$ denote the differential operators for the $S$ and $u$ equations, so they become

$$
\begin{aligned}
& S_{t}=L^{S} S-\gamma^{-1} u f_{u}(S), \\
& u_{t}=L^{u} u+u\left(f_{u}(S)-k\right) .
\end{aligned}
$$

We make several biologically reasonable assumptions concerning the functions $G, f_{u}, f_{w}$. Recalling that the occupation fraction $W=w / w_{\infty} \in[0,1]$, we assume that $G:[0,1] \rightarrow[0,1)$ is $C^{1}$ with $G^{\prime}<0$. In addition, it is assumed that $G(W)=$ $(1-W) g(W)$, where $g \geq 0$ and $g^{\prime}>0$. For example, Freter [18, 19] employs

$$
G(W)=\frac{1-W}{1.1-W}
$$

As usual, $f_{u}, f_{w}$ are $C^{1}$ functions, vanishing at zero and $f_{u}^{\prime}, f_{w}^{\prime} \geq 0$. We further assume that these functions are dominated by linear functions: there exists $p>0$ such that for $f=f_{w}, f_{u}$ we have $f^{\prime}(S) \leq p$.

The existence of a weak solution to our system is guaranteed by the following result, proved in our second appendix.

THEOREM 2.1. There exists a unique weak solution $(S, u, w)$ of $(2.1)-(2.7)$ defined for $t \geq 0$ and it satisfies the range conditions (2.8). Moreover, $S$, $u$ are Hölder continuous uniformly on $\bar{\Omega} \times\left[T^{\prime}, T\right]$ for each $0<T^{\prime}<T$.

The wall-attached bacteria can be removed from the model by setting $\alpha=w=0$. The result is a model of (planktonic) bacterial growth in the flow reactor. It consists of (2.1), (2.3), (2.4), (2.5), (2.7) together with Neumann boundary conditions on $r=R$ :

$$
u_{r}=S_{r}=0, \quad r=R .
$$

This special case, the no wall growth model, is important for comparison purposes.

3. Survival or washout. Our goal in this section is to establish that the bacteria in the flow reactor either are washed out of the reactor (in infinite time) or survive in the sense that the planktonic and wall-attached populations approach a spatially inhomogeneous steady state. A useful technique in our analysis is to multiply the equations by eigenfunctions of an associated linear elliptic eigenvalue problem and integrate over the domain to obtain ordinary differential equations (or inequalities) for these averaged quantities. We use this technique now to show that bacterial growth in the flow reactor is limited by the influx of fresh nutrients, following the argument used in [5]. The adjoint operator to $L^{S}\left(L^{u}\right)$ with homogeneous boundary conditions 
(2.4) with $S^{0}=0,(2.5)$, and radial boundary condition $S_{r}=0$ is denoted by $L_{S}\left(L_{u}\right)$. $L_{S}$ is given by

$$
L_{S} \phi=d_{x}^{S} \phi_{x x}+d_{r}^{S}\left[\phi_{y y}+\phi_{z z}\right]+v(r) \phi_{x}
$$

with homogeneous boundary conditions

$$
\begin{aligned}
& 0=d_{x}^{S} \phi_{x}+v(r) \phi, \quad x=L, \\
& 0=\phi_{x}, \quad x=0, \\
& 0=\phi_{r}, \quad r=R .
\end{aligned}
$$

It's as if the flow through the reactor changes from left to right to right to left. Denote by $-\lambda^{S}$ the principal eigenvalue of the eigenvalue problem

$$
L_{S} \phi=\lambda \phi,
$$

together with the above boundary conditions. Then $-\lambda^{S}<0$, and the corresponding eigenfunction $\phi$ satisfies $\phi>0$ and on $\bar{\Omega}$ and can be normalized by assuming that it attains a maximum of unity (see Theorem 6.3). Let $-\lambda^{u}<0$ be the principal eigenvalue of $L_{u}$ subject to analogous homogeneous boundary conditions and let $\psi>0$ be a corresponding eigenvector normalized so that $\psi \leq \phi$ with equality holding at some point.

THEOREM 3.1. We have the estimates

$$
\limsup _{t \rightarrow \infty} S(t, x, y, z) \leq S^{0}
$$

uniformly in $(x, y, z) \in \Omega$, and

$$
\limsup _{t \rightarrow \infty} \int_{\Omega} u \psi d V \leq \frac{2 \pi \gamma S^{0} \int_{0}^{R} r v(r) d r}{\min \left\{\lambda^{S}, \lambda^{u}+k, k_{w}\right\}},
$$

where the numerator is precisely the net flux of nutrient into the reactor across $x=0$.

Proof. In our final appendix, we establish $S \leq \tilde{S}$, where $\tilde{S}$ satisfies $S_{t}=L^{S} S$ with homogeneous radial boundary condition $S_{r}=0$ and (2.4), (2.5) by a simple comparison argument. Furthermore, noting that $S=S^{0}$ is a steady state of this comparison equation, the linearization of which has a dominant negative eigenvalue, we conclude that $\tilde{S} \rightarrow S^{0}$ as $t \rightarrow \infty$ uniformly in $(x, y, z) \in \Omega$. Now define

$$
X=\int_{\Omega} \phi S d V, \quad Y=\int_{\Omega} \psi u d V, \quad Z=\int_{\partial_{r} \Omega} \psi w d A .
$$

Here $\partial_{r} \Omega$ denotes the cylindrical part of the boundary, $r=R, 0 \leq x \leq L$. We note the following, which follows by integration by parts and Green's identities [21] applied to the two dimensional Laplacian in $y, z$ :

$$
\begin{aligned}
\int_{\Omega} \phi L^{S} S d V & =\int_{\Omega} S L_{S} \phi d V-\gamma^{-1} \int_{\partial_{r} \Omega} \phi w f_{w}(S) d A+S^{0} \int_{r \leq R} v(r) \phi(0, y, z) d y d z \\
& =-\lambda^{S} X-\gamma^{-1} \int_{\partial_{r} \Omega} \phi w f_{w}(S) d A+S^{0} \int_{r \leq R} v(r) \phi(0, y, z) d y d z \\
\int_{\Omega} \psi L^{u} u d V & =\int_{\Omega} u L_{u} \psi d V-\int_{\partial_{r} \Omega} \psi\left(\alpha u(1-W)-w\left[f_{w}(S)(1-G(W))+\beta\right]\right) d A \\
& =-\lambda^{u} Y-\int_{\partial_{r} \Omega} \psi\left(\alpha u(1-W)-w\left[f_{w}(S)(1-G(W))+\beta\right]\right) d A .
\end{aligned}
$$


Differentiation of $X, Y, Z$ and using these relations leads to the following:

$$
\begin{aligned}
X^{\prime} & =-\lambda^{S} X-\gamma^{-1} \int_{\Omega} u \phi f_{u}(S) d V-\gamma^{-1} \int_{\partial_{r} \Omega} \phi w f_{w}(S) d A+S^{0} \int_{r \leq R} v(r) \phi(0, y, z) d y d z, \\
Y^{\prime} & =-\lambda^{u} Y+\int_{\Omega} u \psi\left[f_{u}(S)-k\right] d V-\int_{\partial_{r} \Omega} \psi\left(\alpha u(1-W)-w\left[f_{w}(S)(1-G(W))+\beta\right]\right) d A, \\
Z^{\prime} & =-\left(k_{w}+\beta\right) Z+\int_{\partial_{r} \Omega}\left[\psi w f_{w}(S) G(W)+\psi \alpha u(1-W)\right] d A .
\end{aligned}
$$

Let $Q=\gamma X+Y+Z$. Using $\psi \leq \phi \leq 1$, we find that

$$
\begin{aligned}
Q^{\prime}= & -\lambda^{S} \gamma X-\lambda^{u} Y-k_{w} Z-\int_{\Omega} u \phi f_{u}(S) d V-\int_{\partial_{r} \Omega} \phi w f_{w}(S) d A \\
& +\gamma S^{0} \int_{r \leq R} v(r) \phi(0, y, z) d y d z+\int_{\Omega} u \psi\left[f_{u}(S)-k\right] d V+\int_{\partial_{r} \Omega} \psi w f_{w}(S) d A \\
\leq & -\lambda^{S} \gamma X-\left(\lambda^{u}+k\right) Y-k_{w} Z+\gamma S^{0} \int_{r \leq R} v(r) \phi(0, y, z) d y d z \\
\leq & -\min \left\{\lambda^{S}, \lambda^{u}+k, k_{w}\right\} Q+2 \pi \gamma S^{0} \int_{0}^{R} r v(r) d r .
\end{aligned}
$$

Therefore,

$$
\limsup _{t \rightarrow \infty} Q(t) \leq \frac{2 \pi \gamma S^{0} \int_{0}^{R} r v(r) d r}{\min \left\{\lambda^{S}, \lambda^{u}+k, k_{w}\right\}} .
$$

As $W \in[0,1]$ and $0 \leq S \leq \tilde{S} \rightarrow S^{0}$ as $t \rightarrow \infty$ (noted above), the boundedness of these variables is assured. From the above, we learn that

$$
\limsup _{t \rightarrow \infty} \int_{\Omega} u \psi d V \leq \frac{2 \pi \gamma S^{0} \int_{0}^{R} r v(r) d r}{\min \left\{\lambda^{S}, \lambda^{u}+k, k_{w}\right\}} .
$$

Since $\psi>0$ is continuous on $\bar{\Omega}$, this implies the existence of an a priori asymptotic estimate for $\int_{\Omega} u d V$.

REMARK 3.1. For the no wall growth model $(\alpha=w=0)$ described following Theorem 2.1, (3.1) holds with the modification that $k_{w}$ is dropped from the denominator.

As a corollary to the calculation above, we provide sufficient conditions for the bacteria to be washed out of the reactor. Compare Theorem 3.2 in [5].

Proposition 3.2 (asymptotic stability of washout). If

$$
f_{u}\left(S^{0}\right)-k-\lambda^{u}, \quad f_{w}\left(S^{0}\right)-k_{w}<0,
$$

then

$$
\lim _{t \rightarrow \infty}\left(\int_{\Omega} u d V+\int_{\Omega_{r}} w d A\right)=0
$$

Proof. If (3.3) holds, then since $S \leq \tilde{S} \rightarrow S^{0}$ as $t \rightarrow \infty$, uniformly in $x \in \Omega$, there exists $\mu>0$ and $T>0$ such that

$$
f_{u}(S)-k-\lambda^{u}, \quad f_{w}(S)-k_{w}<-\mu
$$


for all $x \in \bar{\Omega}$ and $t>T$. Then, for $t>T$

$$
\begin{aligned}
(Y+Z)^{\prime} & =-\lambda^{u} Y+\int_{\Omega} u \psi\left[f_{u}(S)-k\right] d V+\int_{\partial_{r} \Omega} \psi w\left[f_{w}(S)+\beta\right] d A-\left(k_{w}+\beta\right) Z \\
& \leq-\lambda^{u} Y+\int_{\Omega} u \psi\left[\lambda^{u}-\mu\right] d V+\int_{\partial_{r} \Omega} \psi w\left[k_{w}-\mu+\beta\right] d A-\left(k_{w}+\beta\right) Z \\
& =-\mu(Y+Z) .
\end{aligned}
$$

The assertion follows immediately.

REMARK 3.2. For the no wall growth model $(\alpha=w=0)$, the same proof shows that $\int_{\Omega} u d V \rightarrow 0$ as $t \rightarrow \infty$ if $f_{u}\left(S^{0}\right)-k-\lambda^{u}<0$.

REMARK 3.3. $\lambda^{u}=\frac{L}{V_{\max }} \lambda$ where $-\lambda<0$ is the principal eigenvalue of the scaled $(\bar{x}=x / L, \bar{r}=r / R)$ eigenvalue problem:

$$
\begin{aligned}
\lambda u & =\theta_{x} u_{\bar{x} \bar{x}}-\left(1-\bar{r}^{2}\right) u_{\bar{x}}+\theta_{r} \bar{r}^{-1}\left(\bar{r} u_{\bar{r})_{\bar{r}},}\right. \\
0 & =-\theta_{x} u_{\bar{x}}+\left(1-\bar{r}^{2}\right) u, \quad \bar{x}=0, \\
0 & =u_{\bar{x}}, \quad \bar{x}=1, \\
u_{\bar{r}} & =0, \quad \bar{r}=1,
\end{aligned}
$$

where $\theta_{x}=\left(d_{x}^{u} / L^{2}\right)\left(L / V_{\max }\right)$ and $\theta_{r}=\left(d_{r}^{u} / R^{2}\right)\left(L / V_{\max }\right)$. In the case that $v(r) \equiv$ $V_{\max }(\epsilon=0)$ is constant, the principal eigenvalue $-\lambda$ can be determined by separation of variables. In fact, the principal eigenfunction $\psi$ depends only on $x$ and $-\lambda<0$ is the principal eigenvalue of the eigenvalue problem

$$
\begin{aligned}
\theta_{x} u^{\prime \prime}-u^{\prime} & =\lambda u, \\
\theta_{x} u^{\prime}(0)-u(0) & =0=u^{\prime}(1) .
\end{aligned}
$$

For more information on $\lambda$ see $[6,5]$.

Our aim now is to obtain sharp conditions for linearized stability of the washout equilibrium

$$
S=S^{0}, u=w=0
$$

and to show the existence of a steady state in which $u, w>0$. We call such a steady state a colonization steady state. The variational equation about the washout equilibrium is

$$
\begin{aligned}
S_{t} & =L^{S} S-\gamma^{-1} u f_{u}\left(S^{0}\right), \\
u_{t} & =L^{u} u+u\left[f_{u}\left(S^{0}\right)-k\right], \\
w_{t} & =w\left[f_{w}\left(S^{0}\right) G(0)-k_{w}-\beta\right]+\alpha u,
\end{aligned}
$$

together with boundary conditions at $x=0$,

$$
\begin{aligned}
& 0=-d_{x}^{S} S_{x}+v(r) S, \\
& 0=-d_{x}^{u} u_{x}+v(r) u,
\end{aligned}
$$

at $x=L$,

$$
S_{x}=u_{x}=0,
$$


and radial boundary conditions on $\partial_{r} \Omega$,

$$
\begin{aligned}
& 0=d_{r}^{S} S_{r}+\gamma^{-1} w f_{w}\left(S^{0}\right), \\
& 0=d_{r}^{u} u_{r}+\alpha u-w\left[f_{w}\left(S^{0}\right)(1-G(0))+\beta\right] .
\end{aligned}
$$

The associated eigenvalue problem is

$$
\begin{aligned}
\lambda S & =L^{S} S-\gamma^{-1} u f_{u}\left(S^{0}\right), \\
\lambda u & =L^{u} u+u\left[f_{u}\left(S^{0}\right)-k\right], \\
\lambda w & =w\left[f_{w}\left(S^{0}\right) G(0)-k_{w}-\beta\right]+\alpha u,
\end{aligned}
$$

together with the above boundary conditions.

It will be convenient to have notation for some related eigenvalue problems. Let $-\lambda_{u}<0$ and $-\lambda_{S}<0$ be the principal eigenvalues of differential operators $L^{u}$ and $L^{S}$. See (3.8) and (3.9) below. In fact, $-\lambda_{u}=-\lambda^{u}$ and $-\lambda_{S}=-\lambda^{S}$ are the same as the principal eigenvalues for the corresponding adjoint operators (see [22, Thm. 3.2, p. 137]) introduced earlier.

$$
\begin{aligned}
\lambda u & =L^{u} u, \\
0 & =-d_{x}^{u} u_{x}+v(r) u, \quad x=0, \quad 0=u_{x}, \quad x=L, \\
0 & =u_{r} \text { in } \partial_{r} \Omega,
\end{aligned}
$$

and

$$
\begin{aligned}
\lambda S & =L^{S} S, \\
0 & =-d_{x}^{S} S_{x}+v(r) S, \quad x=0, \quad 0=S_{x}, \quad x=L, \\
0 & =S_{r} \text { in } \partial_{r} \Omega .
\end{aligned}
$$

We observe that an eigenvalue $\lambda$ of (3.9), with corresponding eigenvector $S$, is an eigenvalue of (3.7) with eigenfunction $(S, 0,0)$. In particular, $-\lambda^{S}$ is the largest of these and there is a corresponding positive eigenfunction $\hat{S}$ of (3.9). See Theorem 6.3 in the appendix.

Observe that neither the equations nor the boundary conditions for $u$ and $w$ in (3.7) contain $S$, so we may consider the subsystem

$$
\begin{aligned}
\lambda u & =L^{u} u+u\left[f_{u}\left(S^{0}\right)-k\right], \\
\lambda w & =w\left[f_{w}\left(S^{0}\right) G(0)-k_{w}-\beta\right]+\alpha u
\end{aligned}
$$

together with the boundary conditions for $u$ as above, separately from (3.7).

The following result establishes the existence of a principal eigenvalue. The proof is deferred to an appendix.

Theorem 3.3 (principal eigenvalue). Let $b \equiv f_{w}\left(S^{0}\right) G(0)-k_{w}-\beta$. Then there exists a real simple eigenvalue $\lambda^{*}>b$ of (3.10) satisfying

$$
\begin{array}{cl}
f_{w}\left(S^{0}\right)-k_{w}<\lambda^{*} \leq f_{u}\left(S^{0}\right)-k-\lambda_{u} & \text { if } f_{w}\left(S^{0}\right)-k_{w}<f_{u}\left(S^{0}\right)-k-\lambda_{u}, \\
f_{w}\left(S^{0}\right)-k_{w}=\lambda^{*} & \text { if } f_{w}\left(S^{0}\right)-k_{w}=f_{u}\left(S^{0}\right)-k-\lambda_{u}, \\
f_{u}\left(S^{0}\right)-k-\lambda_{u}<\lambda^{*}<f_{w}\left(S^{0}\right)-k_{w} & \text { if } f_{w}\left(S^{0}\right)-k_{w}>f_{u}\left(S^{0}\right)-k-\lambda_{u} .
\end{array}
$$

Corresponding to eigenvalue $\lambda^{*}$ is an eigenvector $(\bar{u}, \bar{w})$ satisfying $\bar{u}>0$ in $\bar{\Omega}$ and $\bar{w}>0$ in $\partial_{r} \Omega$. If $\lambda$ is any other eigenvalue of (3.10) corresponding to an eigenvector 
$(u, w) \geq 0$, then $\lambda=\lambda^{*}$ and $(u, w)=c(\bar{u}, \bar{w})$ for some $c>0$. If $\lambda^{*}>-\lambda^{S}$, in particular, if $\lambda^{*}>0$, then $\lambda_{p} \equiv \lambda^{*}$ is a simple eigenvalue of (3.7) with corresponding eigenfunction $(\bar{S}, \bar{u}, \bar{w})$, where $\bar{S}<0$ in $\bar{\Omega}$ and $\bar{S}, \bar{u}, \bar{w}$ are axially symmetric, i.e., in cylindrical coordinates $(r, \theta, x), \bar{S}=\bar{S}(r, x)$.

We expect, but have not proved, that the sign of $\lambda^{*}$ determines the stability properties of the washout steady state. However, observe that the hypotheses of Proposition 3.2 implies that $\lambda^{*}<0$. Below, we will show the existence of a population steady state when $\lambda^{*}>0$.

REMARK 3.4. For the no wall model $(\alpha=w=0)$, the principal eigenvalue becomes $\lambda^{*}=f_{u}\left(S^{0}\right)-k-\lambda_{u}$. Observe that this is one of the bounds on $\lambda^{*}$ in Theorem 3.3 .

The significance of the signs of the components of the eigenfunction is that a bifurcation analysis about the washout steady state would lead to an expansion in small parameters

$$
\begin{aligned}
S & \approx S^{0}+\epsilon \bar{S}, \\
u & \approx \epsilon \bar{u}, \\
w & \approx \epsilon \bar{w}
\end{aligned}
$$

for the colonization steady state. Hence, $S<S^{0}$ and $u, w>0$ as expected. The symmetry of the eigenfunctions suggests that the same symmetry is inherited by the colonization steady state. We will not employ bifurcation techniques here.

A deeper study of the linearized operator requires that its full spectrum be studied. Let $X=C(\bar{\Omega}) \times C(\bar{\Omega}) \times C\left(\partial_{r} \Omega\right)$ be the Banach space with uniform norm. For the domain, $D(\mathcal{L})$, of the linearized operator $\mathcal{L}$, we tentatively take those $(S, u, w) \in X$ such that $S, u$ and their first derivatives are uniformly Hölder continuous on $\bar{\Omega}, S, u$ belong to $C^{2}(\Omega)$ and satisfy appropriate homogeneous Danckwerts' boundary conditions at $x=0, L$ (see above), and $S, u, w$ satisfy radial boundary conditions (3.6). Observe that the coupling of $S, u, w$ through these radial boundary conditions appears as a constraint in defining $D(\mathcal{L})$. Operator $\mathcal{L}$ is then defined on $D(\mathcal{L})$ by

$$
\mathcal{L}(S, u, w)=\left(L^{S} S-\gamma^{-1} u f_{u}\left(S^{0}\right), L^{u} u+a u, b w+\alpha u\right),
$$

where $a=f_{u}\left(S^{0}\right)-k$ and $b$ as in Theorem 3.3. It is not difficult to see that $\mathcal{L}$ is closeable and we rename $\mathcal{L}$ to be the closure of $\mathcal{L}$ and rename $D(\mathcal{L})$ its corresponding domain. Our next result, proved in an appendix, establishes the dominance of the principal eigenvalue over the remaining part of the spectrum.

Proposition 3.4. If $\lambda^{*}>-\lambda^{S}$, then $\Re(\lambda) \leq \lambda^{*}$ for all points $\lambda$ belonging to the spectrum of $\mathcal{L}$. If the reverse inequality holds, then $\Re(\lambda) \leq-\lambda^{S}<0$ for all points $\lambda$ belonging to the spectrum of $\mathcal{L}$.

We now turn to the problem of finding a positive colonization steady state. The equations for a steady state are

$$
\begin{aligned}
& 0=L^{S} S-\gamma^{-1} u f_{u}(S), \\
& 0=L^{u} u+u\left[f_{u}(S)-k\right] \quad \text { in } \Omega, \\
& 0=w\left[f_{w}(S) G(W)-k_{w}-\beta\right]+\alpha u(1-W) \text { in } \partial_{r} \Omega,
\end{aligned}
$$

with boundary conditions (2.4)-(2.6). Our main result is the following (compare Theorem 4.1 in [5]).

THEOREM 3.5. Let $\lambda^{*}>0$ and

$$
b=f_{w}\left(S^{0}\right) G(0)-k_{w}-\beta \neq 0 .
$$


Then there exists a radially symmetric steady state solution $(S, u, w)$ of (3.11) satisfying (in cylindrical coordinates)

$$
0<S(x, r) \leq S^{0}, \quad u(x, r)>0, \text { and } 0<w(x) \leq w_{\infty} .
$$

REMARK 3.5. For the no wall growth model $(\alpha=w=0)$, the conclusion of Theorem 3.5, except for assertions concerning $w$, holds if $\lambda^{*}=f_{u}\left(S^{0}\right)-k-\lambda_{u}>0$. Hypothesis (3.12) is not required in this case.

We are unable to determine the stability properties of a population steady state. However, following Theorem 5.2 in [5], we can at least establish a relatively weak persistence result for the population.

THEOREM 3.6. If

$$
f_{u}\left(S^{0}\right)-k-\lambda_{u}>0 \text { and } f_{w}\left(S^{0}\right)-k_{w}>0,
$$

then there exists $\delta>0$, independent of initial data $\left(S_{0}, u_{0}, w_{0}\right)$ provided that $u_{0}$ and $w_{0}$ do not both vanish identically on their respective domains, such that

$$
\limsup _{t \rightarrow \infty}\left[\sup _{\Omega} u+\sup _{\partial_{r} \Omega} w\right]>\delta .
$$

The proof is given in the appendix on the parabolic problem. This result is hardly optimal. In view of Remark 3.4, the hypotheses require that the washout state is unstable in the case of no wall growth and that the net wall-attached bacterial growth rate is positive.

REMARK 3.6. For the no wall growth model $(\alpha=w=0)$, the conclusion of Theorem 3.6 is modified only by dropping $\sup _{\partial_{r} \Omega} w$ from the inequality, provided $f_{u}\left(S^{0}\right)-k-\lambda_{u}>0$.

It is not difficult to show that $\lambda^{*}$ is monotone increasing in $S^{0}$, for example. If, as we expect (but have not proved), the washout steady state is globally attracting when $\lambda^{*}<0$ and the population steady state is globally attracting when $\lambda^{*}>0$, then there is a threshold value of substrate concentration below which washout occurs and above which the organism survives. Proposition 3.2 and Theorem 3.6 provide some support for this conjecture.

4. Simulations. In this section we describe the numerical algorithm used to approximate (2.1), (2.3), and the associated boundary conditions. In addition, we present a numerical study which augments our analytical results. Throughout this section and in all our numerical experiments, we assume that $w_{\max }=1$ so $w=W$.

Finding a stable spatial and temporal discretization is challenging in this case due in part to the nonlinear boundary conditions and to the physical constraint $S, u$, and $w$ are nonnegative quantities. We assume the solution is radially symmetric, and we take the physical domain to be the rectangle $\{(x, r): 0 \leq x \leq L, 0 \leq r \leq R\}$. This creates a new, artificial boundary condition at $r=0$. However, the symmetry implies that at $r=0$

$$
\left.\frac{\partial S}{\partial r}\right|_{r=0}=\left.\frac{\partial u}{\partial r}\right|_{r=0}=0 .
$$

Thus, the appearance of $1 / r$ in the governing equations is not an issue since the numerical scheme is iterated on $0<r<R$. All of the terms in (2.1) and (2.3) involving spatial derivatives are approximated using standard centered, second-order accurate differencing. 
The temporal differencing uses a forward Euler time integration. Since we are interested mainly in steady states, the low-order accuracy of this method is not an issue. In addition, the diffusion constants are sufficiently small so that the time step in not diffusionally limited. The time step depends only on the grid spacing and the velocity.

Next we examine the boundary conditions. Two of the numerically simplest boundary conditions occur at $x=L$ and are homogeneous Neumann boundary conditions given in (2.5). Applying a uniform mesh in the $x$ and $r$ directions, $S_{i, j}$ approximates the true nodal value of $S$ at the point $\left(x_{i}, r_{j}\right)=(i \Delta x, j \Delta r), 2 \leq j \leq N$, $2 \leq j \leq M$. The boundary requires $i=1, i=N+1, j=1$, or $j=M+1$. A simple application of Taylor's theorem reveals the one-sided, second-order accurate differencing

$$
\left.\Delta x \frac{\partial S}{\partial x}\right|_{x=L} \approx 1.5 S_{N+1, j}-2 S_{N, j}+.5 S_{N-1, j} .
$$

We want this derivative to be zero on the boundary, so we force (4.2) to be zero. Solving for $S_{N+1, j}$, we have an approximation for $S$ on the boundary $x=L$. The quantity $u$ on the boundary $x=L$ is handled in a similar way. The above approximation procedure is also applied to the artificial boundary conditions (4.1) at $r=0$.

The Robin boundary conditions at $x=0$ are treated in a similar way. Indeed, using Taylor's theorem, we obtain the following second-order accurate differencing

$$
\left.\Delta x \frac{\partial S}{\partial x}\right|_{x=0} \approx-1.5 S_{1, j}+2 S_{2, j}-.5 S_{3, j} .
$$

Using the above approximation in (2.4), we find

$$
S_{1, j}=\frac{2 S_{2, j}-.5 S_{3, j}+S^{0} v_{j} \frac{\Delta x}{d_{x}^{S}}}{1.5+v_{j} \frac{\Delta x}{d_{x}^{S}}}
$$

Similarly, the approximation for $u$ on the boundary $x=0$ is given by

$$
u_{1, j}=\frac{2 u_{2, j}-.5 u_{3, j}}{1.5+v_{j} \frac{\Delta x}{d_{x}^{u}}} .
$$

The Robin boundary condition for $u$ at $r=R$ is linear in $u$, and we apply a one-sided difference, similar to (4.3), to find

$$
u_{i, M+1}=\frac{2 u_{i, M}-.5 u_{i, M-1}+w_{i}\left[f_{w}\left(S_{i, M+1}\right)\left(1-G\left(w_{i}\right)\right)+\beta\right] \frac{\Delta r}{d_{x}^{u}}}{1.5+\alpha\left(1-w_{i}\right) \frac{\Delta r}{d_{x}^{u}}} .
$$

If the scheme is iterated on the interior of the rectangle first, all of the above quantities may be evaluated at the new time.

Finally, the boundary condition for $S$ at $r=R$ is the most complicated. In continuous form it is represented by the equation

$$
0=d_{r}^{S} S_{r}+\gamma^{-1} w f_{w}(S) .
$$

The above condition is nonlinear in $S$ when the function $f_{w}(\cdot)$ is nonlinear. One might expect that evaluating $f_{w}(\cdot)$ at the previous time step and using the one-sided, secondorder differencing procedure described above will work. However, the procedure leads 
to an unstable numerical scheme due to the positivity constraint on $S$, i.e., $S \geq 0$. Hence, a nonlinear system has to be solved. Generally, $f_{w}$ is the Monod function $f(x)=m x /(a+x)$, where $m$ and $a$ are given positive constants, which leads to a polynomial equation in $S_{i, M+1}$ of the form

$$
0=\frac{d_{r}^{S}}{\Delta r}\left(1.5 S_{i, M+1}-2 S_{i, M}+.5 S_{i, M-1}\right)+\gamma^{-1} w_{i} f_{w}\left(S_{i, M+1}\right) .
$$

The root $S_{i, M+1}$ may be found exactly, although a Newton-Raphson scheme is more convenient to implement.

Next we turn to a set of numerical experiments. In all simulations we take $f_{u}(S)=$ $f_{w}(S)=m S /(a+S)$, with $m=1.66$ and $a=0.01$, and $\gamma=0.5$, so the Monod function acts almost like a Heaviside function. Since a molecule diffuses more quickly than a bacterial cell in random motion, we take $d^{u} / d^{S}=10^{-1}$, where the diffusion constants satisfy $d_{x}^{u}=d_{r}^{u}=0.001$ and $d_{x}^{S}=d_{r}^{S}=0.01$. The rate of sloughing of wall-attached bacteria into the fluid, $\beta$, and the cell death rate in the wall environment, $k_{w}$, are set equal to each other: $\beta=k_{w}=0.01, G(w)=(1-w) /(1.1-w)$, and $S^{0}=S_{0}$ throughout the numerical studies. The Poisseau flow velocity is given by

$$
v(r)=V_{\max }\left[1-(r / R)^{2}\right],
$$

where we chose $V_{\max }=0.1$. The tube length is fixed at $L=4$, and the radius is fixed at $R=1$. We discretize the domain using a uniform grid with $N=200$ and $M=50$ so that $\Delta x=\Delta r=1 / 50$. The initial conditions for wall-attached bacteria and planktonic bacteria, $w_{0}=0$ and $u_{0}=0.4$, respectively, are the same throughout the numerical studies.

The numerical results presented here were chosen to make the following points: (1) there is a threshold value of supplied nutrient concentration $S^{0}$ below which washout occurs and above which the population survives; (2) this threshold concentration is lower with wall growth than without wall growth $(\alpha=w=0)$; and (3) an antibiotic, introduced in an attempt to eradicate bacteria, which kills planktonic cells but is unable to penetrate the biofilm, is successful in the no wall growth model but not with wall growth (see the discussion below for an amplification of these remarks).

In the first numerical experiment, Figure 4.1(a), we set $S^{0}=8.5 \times 10^{-5}$ (low), $k=0.01$, and $\alpha=0.005$. This results in $u, w \rightarrow 0$ as $t \rightarrow \infty$ as $S^{0}$ is too low (subthreshold). In the second experiment, Figure 4.1(b), Figure 4.2, Figure 4.3, $S^{0}=$ $9.5 \times 10^{-5}$ (moderate), $k=0.01$, and (wall growth) $\alpha=0.005$. Now, the population survives as sufficient nutrient is present (superthreshold). For comparison purposes, the next experiments consider the no wall growth model $(\alpha=w=0)$. In the third numerical experiment, Figure 4.1(c), $S^{0}=9.5 \times 10^{-5}$ (moderate), $k=0.01$ as in the previous experiment, but (no wall growth) $\alpha=0$. Washout occurs, $u \rightarrow 0$ as $t \rightarrow \infty$, indicating that the moderate feed supply is subthreshold in the no wall growth case. In the fourth numerical experiment, Figure 4.1(d), Figure 4.5, we increase $S^{0}=4 \times 10^{-4}$ (high), $k=0.01$, and (no wall growth) $\alpha=0$. Planktonic bacteria survive now because there is sufficient nutrient but note that the threshold value of $S^{0}$ for survival is larger in the no wall growth case than with wall growth. Our next experiments explore the sensitivity of $\lambda^{*}$ to a change in planktonic cell death rate, first for the no wall growth case and then with wall growth. The larger death rate for planktonic bacteria can be viewed as reflecting the presence of an antibiotic. In the fifth numerical experiment, Figure 4.1(e), $S^{0}=4 \times 10^{-4}$ (high) as before but we increase $k$ to $k=0.015$. With no wall growth $(\alpha=0)$, the result is the elimination of the bacteria, $u \rightarrow 0$ as $t \rightarrow \infty$. 


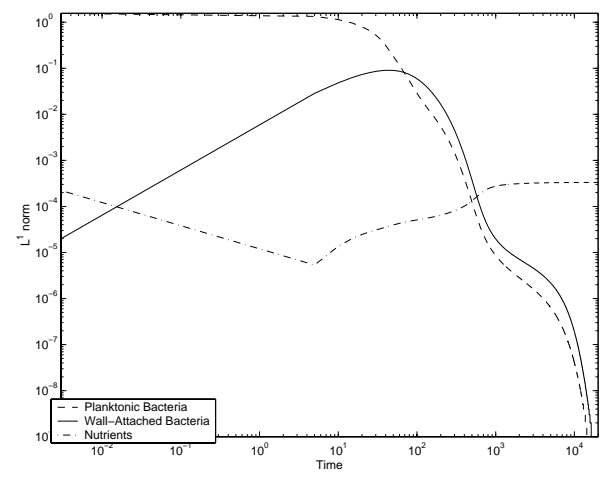

(a) $S^{0}=8.5 \times 10^{-5}, \alpha=0.005, k=0.01$

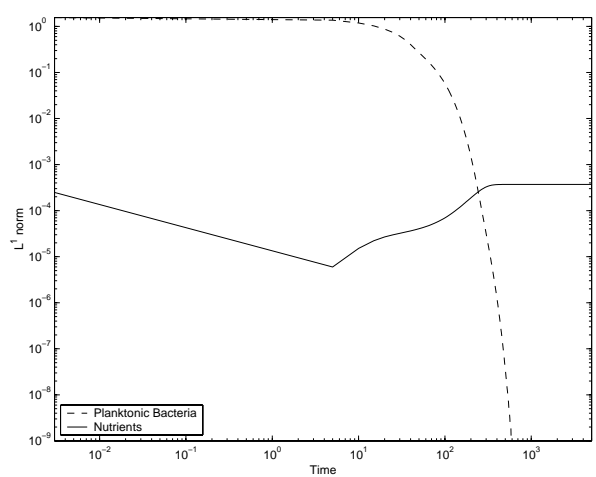

(c) $S^{0}=9.5 \times 10^{-5}, \alpha=0, k=0.01$

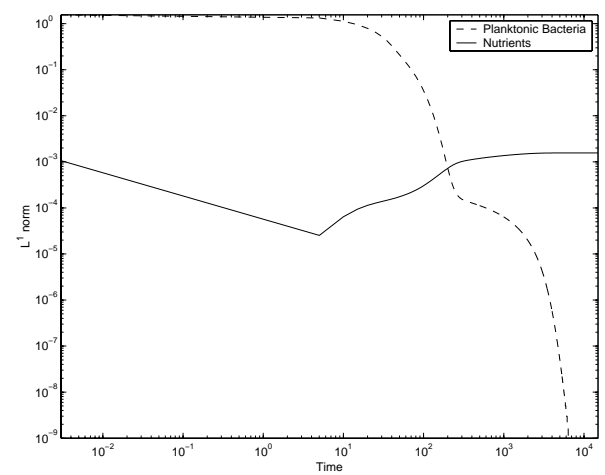

(e) $S^{0}=4 \times 10^{-4}, \alpha=0, k=0.015$

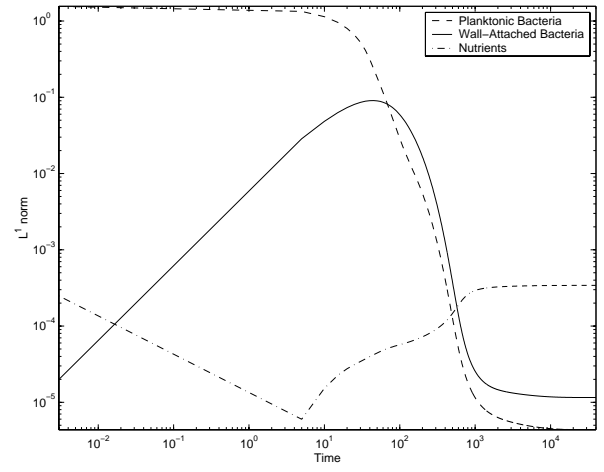

(b) $S^{0}=9.5 \times 10^{-5}, \alpha=0.005, k=0.01$

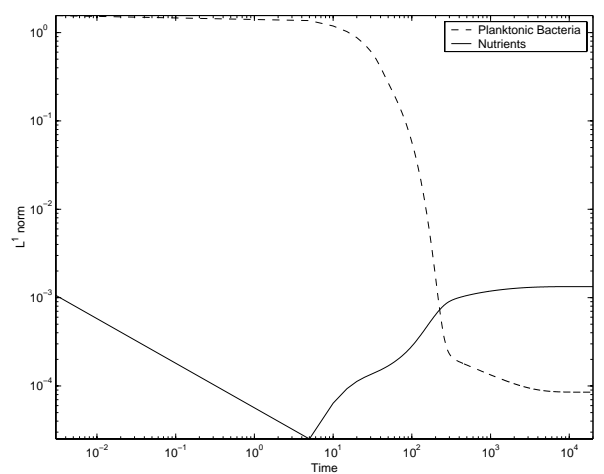

(d) $S^{0}=4 \times 10^{-4}, \alpha=0, k=0.01$

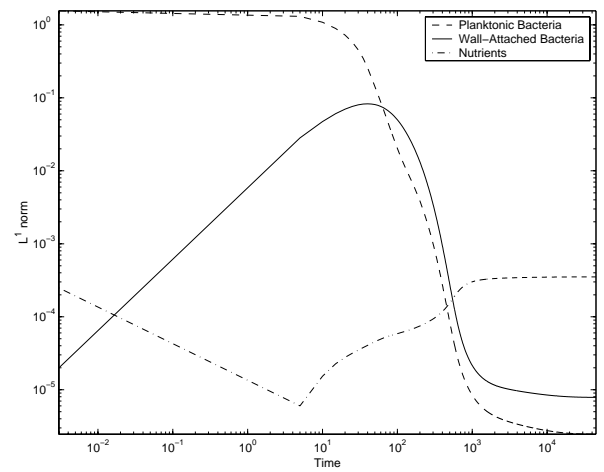

(f) $S^{0}=9.5 \times 10^{-5}, \alpha=0.005, k=0.015$

FIG. 4.1. Log-log plots of the $L^{1}$ norms of densities of the planktonic biomass, $u$, the wallattached cells, $w$, and the nutrient, $S$, versus time.

By contrast, in the sixth numerical experiment, Figure 4.1(f), Figure 4.6, Figure 4.4, with wall growth $(\alpha=0.005)$, moderate $S^{0}=9.5 \times 10^{-5}$, but the larger $k=0.015$ does not result in washout. 

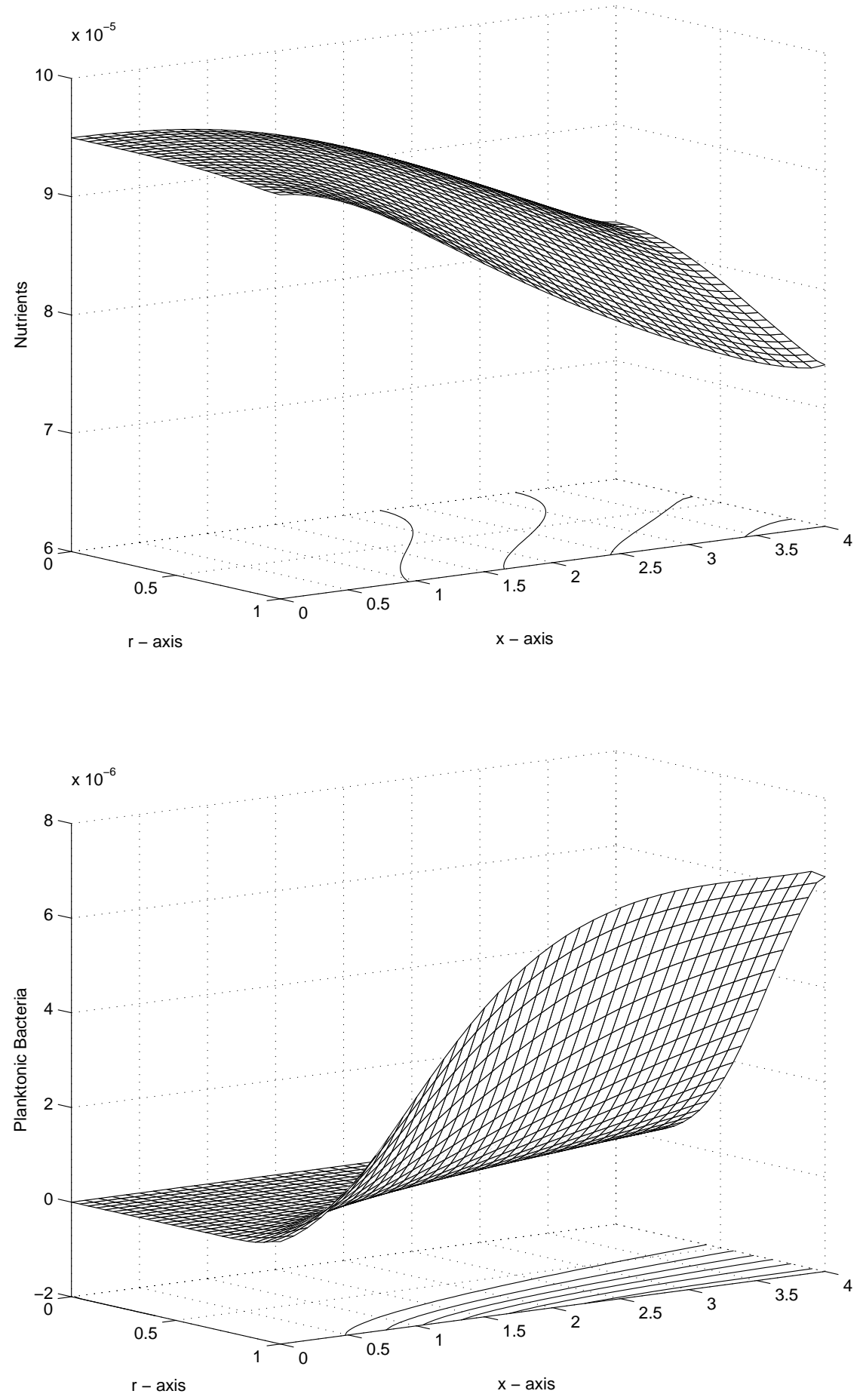

FIG. 4.2. Surface plots of the nutrient density $S$ (top) and the planktonic biomass density $u$ (bottom) at a steady state for $S^{0}=9.5 \times 10^{-5}, \alpha=0.005$, and $k=0.01$. 


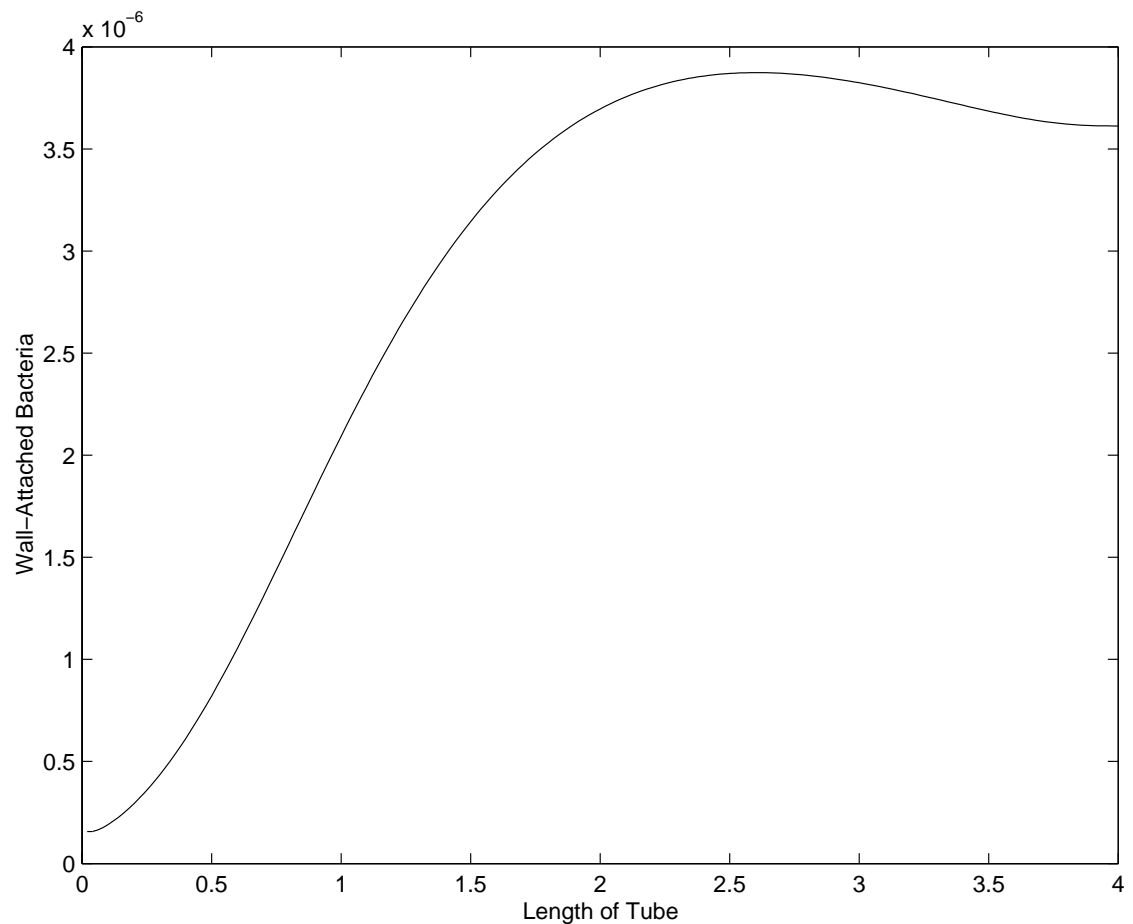

FIG. 4.3. Plot of the areal density of wall-attached cells $w$ at a steady state for $S^{0}=9.5 \times 10^{-5}$, $\alpha=0.005$, and $k=0.01$.

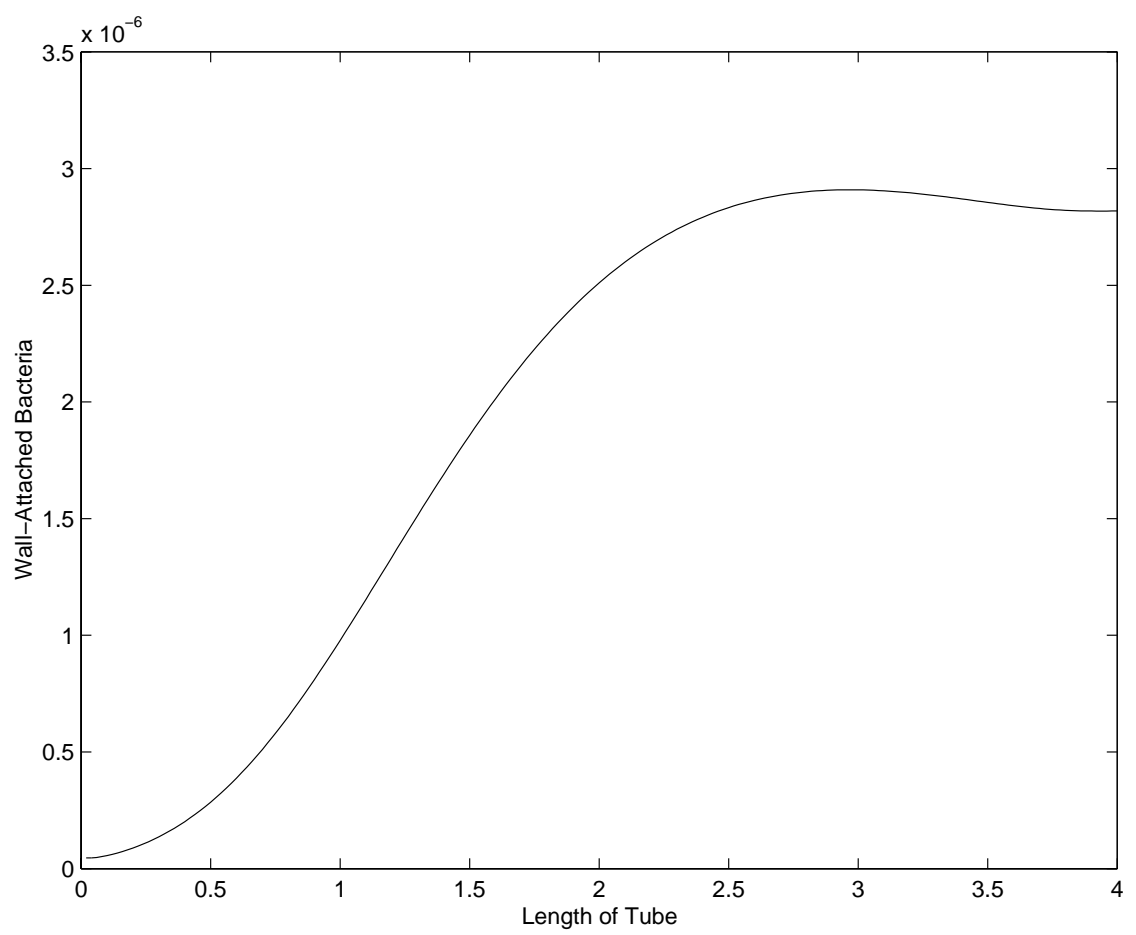

FIG. 4.4. Plot of the areal density of wall-attached cells $w$ at a steady state for $S^{0}=9.5 \times 10^{-5}$, $\alpha=0.005$, and $k=0.015$. 

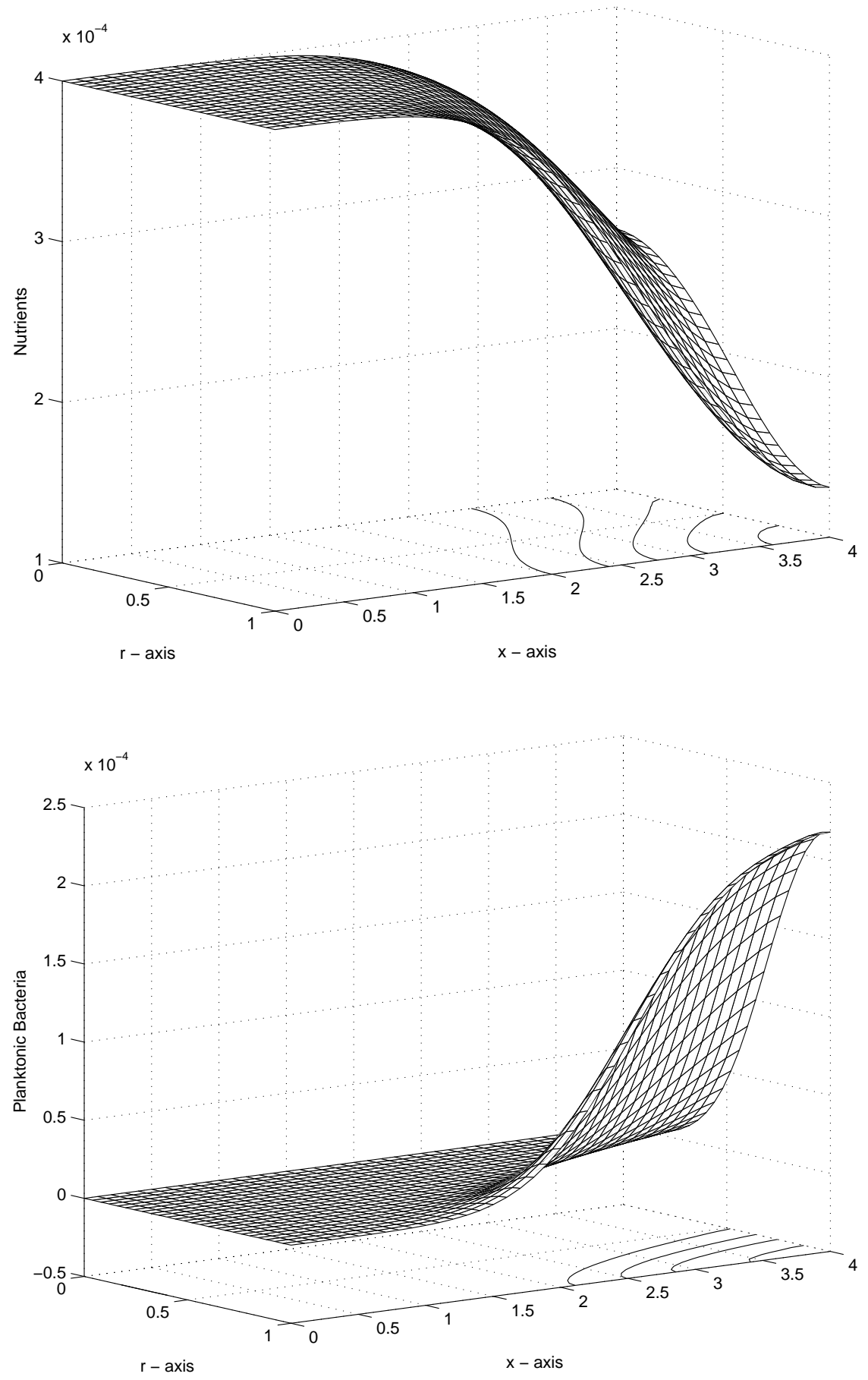

FIG. 4.5. Surface plots of nutrient density $S$ (top) and planktonic biomass density $u$ (bottom) at a steady state for $S^{0}=4 \times 10^{-4}, \alpha=0$ (no wall growth), and $k=0.01$. 

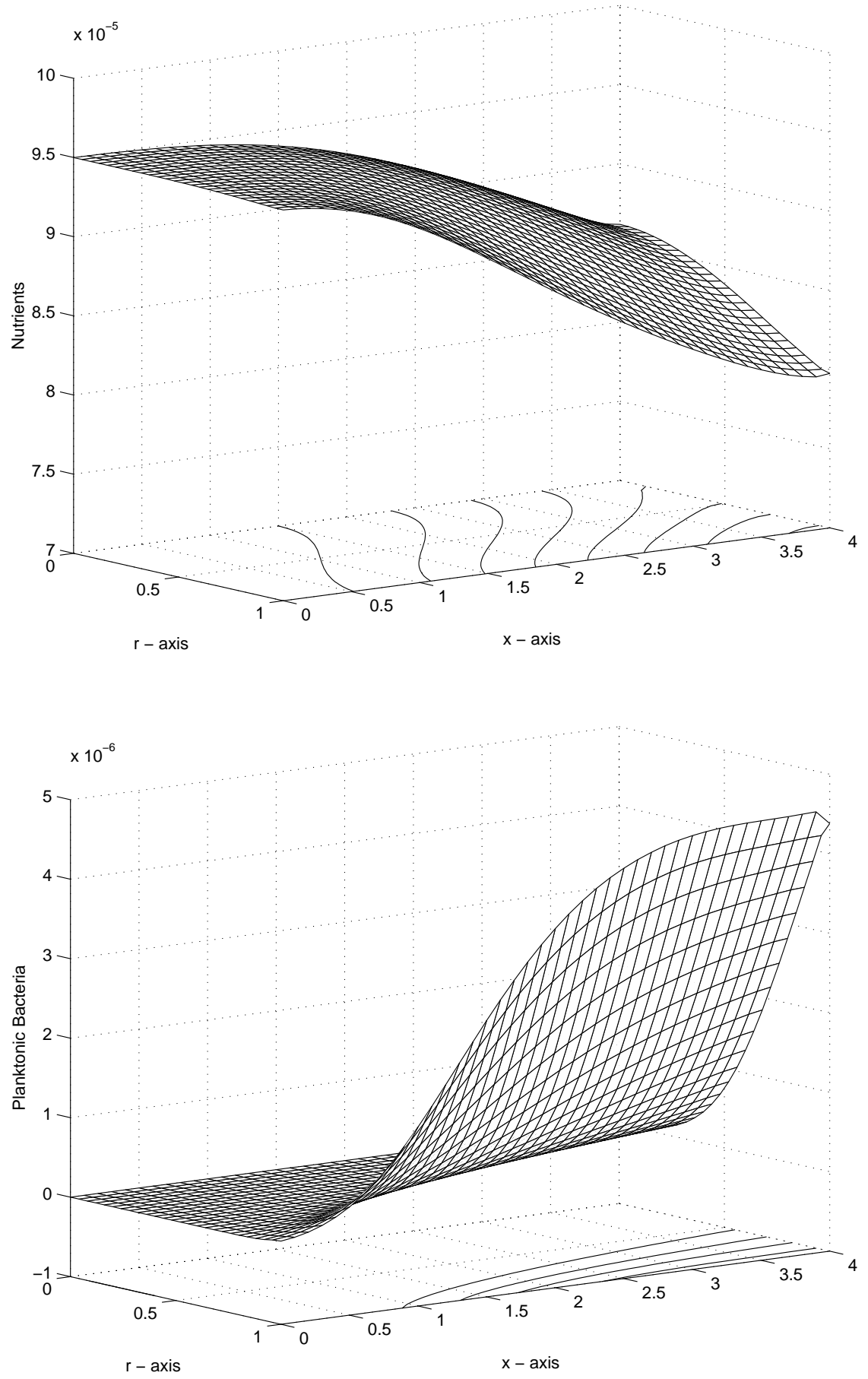

FIG. 4.6. Surface plots of nutrient density $S$ (top) and planktonic biomass density $u$ (bottom) at a steady state for $S^{0}=9.5 \times 10^{-5}, \alpha=0.005$, and $k=0.015$. 
We view Figures $4.2-4.6$ as steady state profiles although actually they are a snapshot, at a very large time, of the dynamics. The salient features of the steady state profiles for the case of wall growth are that planktonic bacterial densities are highest near the outer edge of the cylinder $(r=R)$ where fluid velocity is minimal and near the peak density of wall adherent bacteria. We expect that planktonic bacterial are castoffs from the wall which have not yet washed out. That is, wall adherent bacteria grow fast enough to slough bacteria into the fluid at a rate matching their washout.

5. Discussion. We have investigated mathematically and through numerical simulations a model of a bacterial community consisting of planktonic cells and wallattached cells in a cylindrical flow reactor with Poisseau flow of medium and substrate. Planktonic bacteria are assumed to follow a random run and tumble motion which can be modeled by diffusion $[9,6]$. Wall-attached cells are assumed to be immobile. The layer of bacteria (biofilm) on the cylindrical surface is assumed to be infinitesimally thin so as to have no effect on the fluid flow. This idealization, together with the simple, nonturbulent flow allow a fairly complete mathematical analysis.

Our mathematical analysis and numerical simulations strongly suggest that there are only two dynamical regimes for bacteria in the flow reactor-washout or survival. If, for example, we increase the supplied substrate $S^{0}$ from a low level to a higher level, we find a threshold level of substrate below which bacteria are washed out of the reactor and above which the bacteria successfully colonize the wall and fluid environments. The mechanism for this threshold behavior is intuitively clear: bacterial growth must exceed the outflow of planktonic bacteria at $x=L$ and cell death. Recall that even wall-attached bacteria eventually die $\left(k_{w}>0\right)$ or are sloughed off the wall $(\beta>0)$.

The linearized stability of the washout state is shown to be determined by the sign of a principal eigenvalue $\lambda^{*}$. While we conjecture that the washout state is globally stable when $\lambda^{*}<0$ and that a unique population steady state is globally attractive when $\lambda^{*}>0$ (and $u_{0}, w_{0}$ do not both vanish), we have not proved this. Our results for the no wall growth model are, however, quite sharp. In this case, the washout state is proved globally attracting if $\lambda^{*}<0$ and a nontrivial population steady state exists if $\lambda^{*}>0$. In the case of wall growth, we prove global stability of the washout state when both (see Remark 3.3 for $\lambda$ )

$$
f_{w}\left(S^{0}\right)-k_{w}<0
$$

and

$$
f_{u}\left(S^{0}\right)-k-\frac{L}{V_{\max }} \lambda<0,
$$

which, in turn, implies $\lambda^{*}<0$. The washout steady state is unstable in the linear approximation and a population steady state is proved to exist if $\lambda^{*}>0$. This holds if (compare with (3.8) and (3.9) of [5]) both

$$
f_{w}\left(S^{0}\right)-k_{w}>0
$$

and

$$
f_{u}\left(S^{0}\right)-k-\frac{L}{V_{\max }} \lambda>0
$$

hold, or if

$$
f_{w}\left(S^{0}\right) G(0)-k_{w}-\beta>0 .
$$


In view of Remark 3.4, (5.2) is precisely the linearized instability of the washout state in the case of no wall growth. Condition (5.2) requires growth to exceed the combination of cell death and dilution. This condition alone does not guarantee survival with wall growth since planktonic cells are attracted to the wall which could be a sink if (5.1) fails. Inequality (5.3) says that adherent bacteria grow fast enough on the wall to overcome cell death and sloughing. Finally, in the event that (5.1) and (5.2) hold, we prove that the population persists. This means that there is a lower bound for the sum of the supremum norms of $u$ and $w$, independent of nontrivial initial data, which must ultimately be exceeded.

Comparing the wall growth model with the no wall growth model, we see that bacteria on the wall need only grow fast enough to overcome sloughing and cell death to ensure survival whereas for the no wall growth model, planktonic bacteria must grow fast enough to compensate for cell death and washout. The latter is likely to be a far more severe requirement.

The model is obviously parameter-rich and furthermore, there are a number of important time scales operating. The characteristic time for the flow is $L / V_{\max }$, of diffusion of substrate is $L^{2} / d_{x}^{S}$, of cell motility is $L^{2} / d_{x}^{u}$ and the radial analogues of these. The doubling time for bacteria can be estimated as $\log 2 /\left(f_{w}\left(S^{0}\right)-k_{w}\right)$. Distinct behaviors of model dynamics are to be expected as the relative values of these time scales change to a significant degree. In our simulations, advection dominates, diffusion is relatively insignificant, and doubling time is a little more than twice $L / V_{\max }$.

The lack of effectiveness of antibiotics in controlling bacteria in biofilms has been noted in the literature [11, 31]. As observed by Costerton, Stewart, and Greenberg [11], this resistance to antimicrobials is likely to have multiple causes but one possible mechanism is "the failure of an agent to penetrate the full depth of the biofilm. Polymeric substances like those that make up the matrix of a biofilm are known to retard the diffusion of antibiotics..." An extensive discussion may be found in [31]. In order to explore the phenomena in more depth, we examine the extreme case of a biofilm layer which is impenetrable to an antibiotic $A$ introduced into the flow at $x=0$ at concentration $A^{0}$. We assume that the antibiotic increases cell death rates as its concentration increases: the death rate of planktonic bacteria $k=k(A)$ is an increasing function of $A$. Wall-attached bacteria are unaffected because we assume that the antibiotic cannot penetrate the biofilm layer $\left(A_{r}=0\right.$ when $r=R$; see below). Assuming that the antibiotic is not significantly depleted by its action, its concentration in the flow reactor, $A=A(x, y, z, t)$, satisfies

$$
\begin{aligned}
A_{t} & =L^{A} A, \\
0 & =d_{r}^{A} A_{r}, \quad r=R, \\
v(r) A^{0} & =-d_{x}^{A} A_{x}+v(r) A, \quad x=0, \\
0 & =A_{x}, \quad x=L .
\end{aligned}
$$

Obviously,

$$
A(x, y, z, t) \rightarrow A^{0}, \quad t \rightarrow \infty,
$$

independent of initial data and therefore the long-term effect of the antibiotic is essentially to adjust the death rate of planktonic bacteria to $k=k\left(A^{0}\right)$. However, it is easy to see that $\lambda^{*}$ is relatively insensitive to parameter $k$. The key eigenvalue $\lambda^{*}$ depends on $k$ but satisfies $f_{w}\left(S^{0}\right) G(0)-k_{w}-\beta<\lambda^{*}$ and the lower bound is 
independent of $k$. If (5.3) holds, then $\lambda^{*}>0$ and the washout steady state is unstable. Therefore, we expect the bacterial population to survive in the reactor regardless of the planktonic cell death rate. Certainly the density of planktonic bacteria may be quite low but one expects that the wall-attached cell density is largely unaffected. Thus we conclude that if (5.3) holds, then introduction of antibiotic into the flow reactor will not be effective in eradicating the bacteria.

This contrasts with the case of no wall growth where the stability of the washout state is determined by the principal eigenvalue $\lambda^{*}=f_{u}\left(S^{0}\right)-k\left(A^{0}\right)-\frac{L}{V_{\max }} \lambda$. See Remark 3.4. Indeed, bacteria are washed out of the reactor when $\lambda^{*}<0$ (see Remark 3.2). It is clearly quite conceivable that when $A^{0}$ is sufficiently large that $\lambda^{*}<0$. Therefore, one expects to be able to eradicate bacteria from the flow reactor in the absence of wall growth but not when wall growth can occur. The numerical experiments contained in Figures 4.1(e) and 4.1(f) support these conclusions.

6. Appendix. Positivity for elliptic equations. In this section we are concerned with extending results of Amann [1] to our setting. The principal difficulty is that the boundary of $\Omega$, and consequently the normal vector to the boundary, are only piecewise smooth. In addition, the coefficient of $u$ in the Robin boundary conditions is only piecewise smooth.

Consider the problem

$$
\begin{aligned}
-L u+c u & =g, \Omega, \\
d_{r} u+\alpha u & =f, \partial_{r} \Omega,
\end{aligned}
$$

with homogeneous Danckwert's boundary conditions at the "caps" $x=0$ and $x=L$. We assume that $c, \alpha \geq 0$ and $L=L^{S}, L^{u}$. The following result may be compared with Theorem 4.2 in [1].

THEOREM 6.1. The boundary value problem (6.1) has a unique classical solution $u \in C^{1+\mu}(\bar{\Omega}) \cap C^{2}(\Omega)$ for each $(g, f) \in C^{\mu}(\bar{\Omega}) \times C^{\mu}\left(\partial_{r} \Omega\right)$. It also has a unique weak solution $u \in C^{\mu}(\bar{\Omega})$ for each $(g, f) \in C(\bar{\Omega}) \times C\left(\partial_{r} \Omega\right)$. There exists $c_{0} \geq 0$ such that for $c \geq c_{0}$, the corresponding weak solution operator $u=K(g, f)$ is a compact linear operator $K: C(\bar{\Omega}) \times C\left(\partial_{r} \Omega\right) \rightarrow C(\bar{\Omega})$. Furthermore, for $c \geq c_{0}, K$ is positive in the sense that $u=K(g, f) \geq 0$ if $g, f \geq 0$. It is also strongly positive: $u>0$ in $\bar{\Omega}$ if $(g, f) \geq 0$ and $(g, f) \neq(0,0)$. Finally, $u$ is radially symmetric if $g$ and $f$ are.

In fact, $c_{0}$ can be taken to be zero as we show later.

Proof. The first assertion follows from Theorem 3.2 in [24] where it is shown that $u \in C^{2}(\Omega) \cap C(\bar{\Omega})$ satisfies an interior Schauder estimate. The latter implies (use (3.7) in [24] and Lemma 2.1 in [20]) that $u \in C^{1+\mu}(\bar{\Omega})$.

Weak solutions $u \in W^{1,2}(\Omega)$ of (6.1) satisfy

$$
\begin{aligned}
& \int_{\Omega} d_{x} u_{x} w_{x}+d_{r}\left(u_{y} w_{y}+u_{z} w_{z}\right)+v(r) u_{x} w+c u w d V \\
&=\int_{\Omega} g w d V-\int_{x=0} v(r) u w d A+\int_{\partial_{r} \Omega} w(f-\alpha u) d A
\end{aligned}
$$

for all $w \in W^{1,2}(\Omega)$. Standard results imply the existence of a unique weak solution (see [21]) for $(g, f) \in L^{2}(\Omega) \times L^{2}\left(\partial_{r} \Omega\right)$ which gives rise to a bounded linear operator $u=K(g, f)$ where

$$
K: L^{2}(\Omega) \times L^{2}\left(\partial_{r} \Omega\right) \rightarrow L^{2}(\Omega)
$$


Lieberman's result, cited above, guarantees that $u=0$ is the only solution of the homogeneous problem $(g, f)=(0,0)$. By Dung [16], we have $u=K(g, f)$ contained in $C^{\mu}(\bar{\Omega})$ if $(g, f) \in C(\bar{\Omega}) \times C\left(\partial_{r} \Omega\right)$ and if $u \in L^{\infty}(\Omega)$.

By Remark 2.1 in [15], there exists $c_{0} \geq 0$ such that for $c \geq c_{0}$ and every $(g, f) \geq 0$, the weak solution $u=K(g, f)$ satisfies $u \geq 0$. Thus, for $c \geq c_{0}, K$ is a positive operator on $C^{\mu}(\bar{\Omega}) \times C^{\mu}\left(\partial_{r} \Omega\right)$. This leads to the conclusion that for $(g, f) \in C^{\mu}(\bar{\Omega}) \times$ $C^{\mu}\left(\partial_{r} \Omega\right)$, we have

$$
\|K(g, f)\|_{C(\bar{\Omega})} \leq C\left[\|g\|_{C(\bar{\Omega})}+\|f\|_{C\left(\partial_{r} \Omega\right)}\right] .
$$

Indeed, let $e_{1} \equiv K(1,0), e_{2} \equiv K(0,1) \in C^{2}(\Omega) \cap C^{1+\mu}(\bar{\Omega})$ and observe that

$$
u=K(g, f) \leq K\left(\|g\|_{C(\bar{\Omega})} 1,\|f\|_{C\left(\partial_{r} \Omega\right)} 1\right)=\|g\|_{C(\bar{\Omega})} e_{1}+\|f\|_{C\left(\partial_{r} \Omega\right)} e_{2}
$$

and, similarly,

$$
u=K(g, f) \geq K\left(-\|g\|_{C(\bar{\Omega})} 1,-\|f\|_{C\left(\partial_{r} \Omega\right)} 1\right)=-\|g\|_{C(\bar{\Omega})} e_{1}-\|f\|_{C\left(\partial_{r} \Omega\right)} e_{2},
$$

so we have the pointwise estimate

$$
|u| \leq\|g\|_{C(\bar{\Omega})} e_{1}+\|f\|_{C\left(\partial_{r} \Omega\right)} e_{2}
$$

which proves (6.3). Thus, as $C^{\mu}(\bar{\Omega}) \times C^{\mu}\left(\partial_{r} \Omega\right)$ is dense in $C(\bar{\Omega}) \times C\left(\partial_{r} \Omega\right)$ and $C(\bar{\Omega})$ continuously imbeds in $L^{2}(\Omega)$, the weak solution operator $K$ on $L^{2}(\Omega) \times L^{2}\left(\partial_{r} \Omega\right)$ restricts to a bounded operator on the continuous function spaces ((6.3) holds) $K$ : $C(\bar{\Omega}) \times C\left(\partial_{r} \Omega\right) \rightarrow C(\bar{\Omega})$. Furthermore, the regularity $u=K(g, f) \in C^{\mu}(\bar{\Omega})$ is retained for $(g, f) \in C(\bar{\Omega}) \times C\left(\partial_{r} \Omega\right)$. Indeed, as our domain satisfies the uniform exterior cone condition, a combination of the arguments in the proof of Theorem 8.29 in [21] and pages 937-938 in [15] shows that the Hölder norm of $u$ on $\bar{\Omega}$ can be majorized by a constant depending on the $L^{\infty}$ norms of $f, g$, and $u$. By (6.3), we then assert that the Hölder norm of $u$ can be majorized by the $L^{\infty}$ norms of $f, g$. Therefore, $K$ maps bounded sets of $C(\bar{\Omega}) \times C\left(\partial_{r} \Omega\right)$ into bounded sets of $C^{\mu}(\bar{\Omega})$, and thus it is compact. As $K$ is the weak solution operator for continuous $(g, f)$, positivity of $K$ holds as noted above. Strong positivity follows from Theorem 4 of [15]. Radial symmetry of $u$, in case $(g, f)$ is, follows from uniqueness and the fact $u \circ R_{\theta}$ is also a solution where $R_{\theta}$ is rotation about the $x$-axis through angle theta.

Consider the nonlinear boundary value problem

$$
\begin{aligned}
-L u+c u & =g(x, y, z, u), \Omega, \\
d_{r} u+\alpha u & =f(x, y, z, u), \partial_{r} \Omega,
\end{aligned}
$$

where $g, f$ are Hölder continuous in all arguments with exponent $\mu$. We show that (6.4) is equivalent to a fixed point problem in $C(\bar{\Omega})$.

TheOREM 6.2. Let $c \geq c_{0}$. The boundary value problem (6.4) is equivalent to the fixed point problem

$$
u=K(G(u), F(u)), \quad u \in C(\bar{\Omega}),
$$

where $G: C(\bar{\Omega}) \rightarrow C(\bar{\Omega})$ and $F: C\left(\partial_{r} \Omega\right) \rightarrow C\left(\partial_{r} \Omega\right)$ are the Nemytskii operators associated with $g$ and $f$.

Proof. If $u$ is a classical solution of (6.4) then it obviously satisfies (6.5) by Theorem 6.1 since $(g, f)=(G(u), F(u)) \in C^{\mu}(\bar{\Omega}) \times C^{\mu}\left(\partial_{r} \Omega\right)$. 
If $u \in C(\bar{\Omega})$ satisfies (6.5), then, since $(G(u), F(u)) \in C(\bar{\Omega}) \times C\left(\partial_{r} \Omega\right)$, we conclude from Theorem 6.1 that $u$ belongs to $C^{\mu}(\bar{\Omega})$. Then, $(G(u), F(u)) \in C^{\mu}(\bar{\Omega}) \times C^{\mu}\left(\partial_{r} \Omega\right)$, so Theorem 6.1 implies that $u$ is a classical solution of (6.4).

Consider the eigenvalue problem

$$
\begin{aligned}
-L u & =\lambda u, \Omega, \\
d_{r} u+\alpha u & =0, \partial_{r} \Omega .
\end{aligned}
$$

The following result is to be compared with Theorem 4.3 in [1].

THEOREM 6.3. The eigenvalue problem (6.6) has a principal eigenvalue $\lambda_{0}$ which is positive. There exists exactly one normalized eigenfunction $u_{0}$ corresponding to $\lambda_{0}$ and it can be chosen to be positive in $\bar{\Omega}$. Any other nonnegative eigenfunction must be scalar multiple of $u_{0}$. If $\lambda$ is any other eigenvalue, then $\Re \lambda>\lambda_{0}$.

Proof. According to Theorem 6.1, (6.6) has no real eigenvalues satisfying $\lambda \leq 0$. We show that the eigenvalues of (6.6) are related to those of the compact, strongly positive linear operator $K_{0} \equiv K(\bullet, 0)$ of Theorem 6.1 corresponding to $c=c_{0}$. Consider the eigenvalue problem

$$
\eta u=K_{0} u .
$$

Obviously, if $u$ is a classical solution of (6.6) corresponding to eigenvalue $\lambda$, then $\lambda>0$ and $u$ is also a solution of (6.7) corresponding to $\eta=\left(\lambda+c_{0}\right)^{-1}$. Conversely, suppose that $u \in C(\bar{\Omega})$ is nontrivial and satisfies (6.7) for some $\eta$. Then $\eta \neq 0$ or else 0 is the weak solution of (6.1) with $g=u \neq 0, f=0$, and $c=c_{0}$, a contradiction. Therefore, $u$ belongs to the range of $K_{0}$ so $u \in C^{\mu}(\bar{\Omega})$ by Theorem 6.1 . But then $u \in C^{1+\mu}(\bar{\Omega}) \cap C^{2}(\Omega)$ by Theorem 6.1 and it is a classical solution of (6.6) corresponding to $\lambda=\eta^{-1}-c_{0}$.

The positivity of $u_{0}$ and other assertions follow from the Krein-Rutman theorem (see, e.g., Theorem in $3.2[1]$ ) since $K_{0}$ is compact and strongly positive.

As an immediate corollary of the proof of Theorem 6.3 we obtain the following.

REMARK 6.1. The constant $c_{0}$ in Theorem 6.1 may be taken to be $c_{0}=0$.

Proof. If $0 \leq c<c_{0}$ in $(6.1),(g, f) \geq(0,0)$, and $(g, f) \neq(0,0)$, then $u$ satisfies $-L u+c_{0} u=\left(c_{0}-c\right) u+g$ and, therefore,

$$
\eta^{-1} u-K_{0} u=w
$$

where $\eta=c_{0}-c>0$ and $w=\eta^{-1} K(g, f)>0$ by Theorem 6.1. By the previous proof, the spectral radius of $K_{0}$ is $\left(\lambda_{0}+c_{0}\right)^{-1}$, where $\lambda_{0}>0$ is the principal eigenvalue of (6.6). As $\eta^{-1}>\left(\lambda_{0}+c_{0}\right)^{-1}$, it follows from Theorem 3.2 of [1] that $u>0$. Therefore, the solution operator for Hölder continuous $(g, f)$ is positive for $0 \leq c<c_{0}$, and the proof of Theorem 6.1 holds in this case.

Finally, we need a counterpart to the existence part of Theorem 4.4 of [1] for the problem

$$
\begin{aligned}
-L u-\lambda u & =g, \Omega, \\
d_{r} u+\alpha u & =f, \partial_{r} \Omega .
\end{aligned}
$$

Theorem 6.4. Let $(g, f) \in C^{\mu}(\bar{\Omega}) \times C^{\mu}\left(\partial_{r} \Omega\right)$. Then, for every $\lambda<\lambda_{0}$, (6.8) has exactly one solution, which is positive in $\bar{\Omega}$ if $(g, f) \geq 0$ and do not both vanish identically.

Proof. If $\lambda \leq 0$, the result is immediate from Theorem 6.1. The remainder of the proof follows exactly as in Theorem 4.4 of [1]. 
7. Appendix. Principal eigenvalue. We first prove Theorem 3.3, then Proposition 3.4 following it.

Proof. We first note that if there exists a real eigenvalue $\lambda^{*}$ of (3.10) with a corresponding eigenvector $(\bar{u}, \bar{w})$ satisfying $\bar{u}>0$ in $\bar{\Omega}$ and $\bar{w}>0$ in $\partial_{r} \Omega$ and if $\lambda^{*}>-\lambda^{S}$, then there exists $\bar{S}<0$ such that $(\bar{S}, \bar{u}, \bar{w})$ is an eigenvector for (3.7) corresponding to $\lambda^{*}$. Indeed, from (3.7), $\bar{S}$ must satisfy

$$
\begin{aligned}
-L^{S} S-\left(-\lambda^{*}\right) S & =-\gamma^{-1} \bar{u} f_{u}\left(S^{0}\right), \\
0 & =-d_{x}^{S} S_{x}+v(r) S, \quad x=0, \quad 0=S_{x}, \quad x=L, \\
d_{r}^{S} S_{r} & =-\gamma^{-1} \bar{w} f_{w}\left(S^{0}\right) \text { in } \partial_{r} \Omega .
\end{aligned}
$$

Since $\lambda^{*}>-\lambda^{S}$, it follows from Theorem 6.4 in the appendix that there is a unique solution $\bar{S}$ of the boundary value problem and further that $\bar{S}<0$. Furthermore, if $(\bar{u}, \bar{w})$ is axially symmetric, then so is $S$ by the uniqueness of the solution of the boundary value problem and by its invariance with respect to the group action $\theta \rightarrow$ $\theta+\phi$ acting on functions.

We now turn to the eigenvalue problem (3.10). It will be convenient to set $a \equiv$ $f_{u}\left(S^{0}\right)-k$ and $c \equiv f_{w}\left(S^{0}\right)(1-G(0))+\beta>0$. Note that $f_{w}\left(S^{0}\right)-k_{w}=b+c$. Let us first show that a positive eigenvector $(u, w)$ cannot correspond to $\lambda=b$. Clearly, this would imply that $u \equiv 0$ on $\partial_{r} \Omega$. We may write the radial boundary conditions for $u$ as $d_{r}^{u} u_{r}+\alpha u=c w \geq 0$ and since $u \geq 0$ is nontrivial, it follows from Theorem 4 in [15] that $u>0$ in $\bar{\Omega}$, a contradiction.

Observe that we may solve the algebraic equation for $w$ to get $w=\frac{\alpha}{\lambda-b} u$. As we desire positivity of $u, w$ we must have $\lambda>b$. Substituting into the radial boundary condition for $u$, we get a single equation for $u$ :

$$
\begin{aligned}
-L^{u} u & =(a-\lambda) u, \\
0 & =-d_{x}^{u} u_{x}+v(r) u, \quad x=0, \quad 0=u_{x}, \quad x=L, \\
0 & =d_{r}^{u} u_{r}+\alpha \frac{\lambda-b-c}{\lambda-b} u, \quad \partial_{r} \Omega .
\end{aligned}
$$

If $\eta=\alpha \frac{\lambda-b-c}{\lambda-b} \geq 0$, i.e., if $\lambda \geq b+c$, then it is natural to consider the eigenvalue problem

$$
\begin{aligned}
\lambda u & =L^{u} u, \\
0 & =-d_{x}^{u} u_{x}+v(r) u, \quad x=0, \quad 0=u_{x}, \quad x=L, \\
0 & =d_{r}^{u} u_{r}+\eta u \text { in } \partial_{r} \Omega .
\end{aligned}
$$

Suppose for some $\lambda_{0} \geq b+c$ we find a function $u>0$ in $\bar{\Omega}$ satisfying (7.1). We may regard $\eta=\alpha \frac{\lambda_{0}-b-c}{\lambda_{0}-b}$ as fixed. By the positivity of $u, \lambda_{0}$ is the principal eigenvalue of this eigenvalue problem, and therefore it is a simple eigenvalue by well-known results (see [1]). Now, if $(\tilde{u}, \tilde{w})$ were any eigenfunction for (3.10) corresponding to the same value $\lambda_{0}$, then $\tilde{u}$ satisfies (7.1), so $\tilde{u}=c u$ for some $c$, implying the simplicity of $\lambda_{0}$ as an eigenvalue of (3.10) in this case.

We will establish the existence of a positive eigenvector for (7.1) corresponding to an eigenvalue $\lambda \geq b+c$ by comparing it with (7.2). For each $\eta \geq 0$, there is a principal eigenvalue $\lambda=\lambda(\eta)<0$ of $(7.2)$, with $\lambda(0)=-\lambda^{u}$. As is well known, this eigenvalue is simple and consequently is a continuous function of $\eta \geq 0$ and there exists a corresponding eigenvector $u=u(\eta)$ which is positive in $\bar{\Omega}$, normalized so that its maximum value is one. See Theorem 6.3. We seek to find a positive eigenvector 
for (3.10) in the form $u=u(\eta)$ and $w=k u(\eta)$ for suitable $k>0$ and $\eta \geq 0$. From (3.10) and the boundary conditions for $u$ on $\partial \Omega_{r}$, it is necessary and sufficient for $(u, w)=(u(\eta), k u(\eta))$ to be an eigenvector so that

$$
\begin{aligned}
\lambda & =\lambda(\eta)+a, \\
\lambda k & =b k+\alpha, \\
0 & =-\eta+\alpha-c k .
\end{aligned}
$$

The first equation gives $\lambda$ and the third determines $k=\frac{\alpha-\eta}{c}>0$ provided $\eta<\alpha$. Inserting these results into the second equation leads to an implicit equation for $\eta \in[0, \alpha)$ :

$$
0=b-a+\frac{\alpha c}{\alpha-\eta}-\lambda(\eta)
$$

A maximum principle argument, given below, shows that $\lambda(\eta)$ is nonincreasing in $\eta \geq$ 0 , so the right-hand side is a strictly increasing, continuous function of $\eta \in[0, \alpha)$ which approaches positive infinity as $\eta$ approaches $\alpha$. Therefore, there is a unique solution $\eta_{0} \in[0, \alpha)$ provided the right-hand side is nonpositive at $\eta=0$. This last condition is just $b-a+c+\lambda^{u} \leq 0$ or $b+c \leq a-\lambda_{u}$ which becomes $f_{w}\left(S^{0}\right)-k_{w} \leq f_{u}\left(S^{0}\right)-k-\lambda^{u}$. If the latter condition holds, then we have found a positive eigenvector $(\bar{u}, \bar{w})$ of $(3.10)$ corresponding to the simple eigenvalue $\lambda^{*} \equiv a+\lambda\left(\eta_{0}\right)$. As $\lambda\left(\eta_{0}\right) \leq \lambda(0)=-\lambda^{u}$, $\lambda^{*} \leq a-\lambda^{u}=f_{u}\left(S^{0}\right)-k-\lambda^{u}$. Also, $\lambda^{*}=a+\lambda\left(\eta_{0}\right)=b+\frac{\alpha c}{\alpha-\eta_{0}} \geq b+c=$ $f_{w}\left(S^{0}\right)-k_{w}$, with strict inequality if $f_{w}\left(S^{0}\right)-k_{w}<f_{u}\left(S^{0}\right)-k-\lambda^{u}$. In summary, if $f_{w}\left(S^{0}\right)-k_{w} \leq f_{u}\left(S^{0}\right)-k-\lambda^{u}$, then $f_{w}\left(S^{0}\right)-k_{w} \leq \lambda^{*} \leq f_{u}\left(S^{0}\right)-k-\lambda^{u}$, with strict inequalities in the latter if strict inequality holds in the former.

Here we establish the claim made in the previous argument that $\lambda(\eta)$ is nonincreasing in $\eta$. Let $0 \leq \eta<\tilde{\eta}$ and $\lambda=\lambda(\eta)$. If $u>0$ is the normalized principal eigenvector for (7.2) corresponding to $\eta$, then

$$
\begin{aligned}
\lambda u & =L^{u} u, \\
0 & =-d_{x}^{u} u_{x}+v(r) u, \quad x=0, \quad 0=u_{x}, \quad x=L, \\
d_{r}^{u} u_{r}+\tilde{\eta} u & =(\tilde{\eta}-\eta) u>0, \quad \partial_{r} \Omega ;
\end{aligned}
$$

hence, using Theorem 17, p. 89 in [28], it is easy to see that $\lambda(\tilde{\eta}) \leq \lambda(\eta)$.

Observe that the principal eigenvector $u$ must be axially symmetric by virtue of the well-known fact that the principal eigenvalue $\lambda(\eta)$ is simple with positive eigenvector $u$. Indeed, as the eigenvalue equation is invariant with respect to a rotation about the $x$-axis, $\tilde{u}=u$ evaluated at the rotated coordinates is again a positive eigenvector and therefore $\tilde{u}=c u$ for some constant $c$. Clearly $c$ must be positive and since rotation does not change the uniform norm, $c=1$.

We now consider (3.10) when $f_{w}\left(S^{0}\right)-k_{w}>f_{u}\left(S^{0}\right)-k-\lambda^{u}$ or $b+c>a-\lambda^{u}$. For $\lambda \neq b,(3.10)$ becomes

$$
\begin{aligned}
-L^{u} u+(\omega-a) u & =(\omega-\lambda) u, \\
d_{r}^{u} u_{r}+\alpha u & =\frac{\alpha c}{\lambda-b} u, \\
w & =\frac{\alpha u}{\lambda-b},
\end{aligned}
$$

and usual boundary conditions at $x=0, L$. We assume that $\omega>b, a$ and we will 
adjust it further later on. This in turn is equivalent to

$$
u=K_{\omega}\left((\omega-\lambda) u, \frac{\alpha c}{\lambda-b} t(u)\right)
$$

where $u=K_{\omega}(f, g)$ is the solution operator for the elliptic problem

$$
\begin{aligned}
-L^{u} u+(\omega-a) u & =f, \\
d_{r}^{u} u_{r}+\alpha u & =g,
\end{aligned}
$$

and $t(u)$ denotes the restriction of $u \in C(\bar{\Omega})$ to $\partial_{r} \Omega$. By Theorem 6.1, $K_{\omega}: C(\bar{\Omega}) \times$ $C\left(\partial_{r} \Omega\right) \rightarrow C(\bar{\Omega})$ is compact and strongly positive: $u>0$ if $u=K_{\omega}(f, g),(f, g) \geq 0$, and $(f, g) \neq(0,0)$. Define

$$
T(u, \lambda)=K_{\omega}\left((\omega-\lambda) u, \frac{\alpha c}{\lambda-b} t(u)\right)
$$

for $\lambda \neq b$. For fixed $\lambda, T(\cdot, \lambda)$ is a compact operator and (7.5) becomes

$$
u=T(u, \lambda) .
$$

If we regard $\lambda$ as a parameter, then we may interpret (7.6) as implying that one is an eigenvalue of the operator $T(\cdot, \lambda)$ with eigenvector $u \neq 0$, in which case $r(\lambda) \geq 1$ where $r(\lambda)=\rho(T(\cdot, \lambda))$ is the spectral radius of $T(\cdot, \lambda)$. Because $K_{\omega}$ is strongly positive, if $b<\lambda<\omega$, then $T(\cdot, \lambda)$ is strongly positive and compact. By the Krein-Rutman theorem, there is an eigenvalue $b<\lambda<\omega$ corresponding to a positive eigenvector $u$ if and only if $r(\lambda)=1$. As $\lambda \rightarrow \omega-\lambda$ and $\lambda \rightarrow \frac{\alpha c}{\lambda-b}$ are strictly decreasing, if $b<\lambda_{1}<\lambda_{2}<\omega$ and $T\left(u, \lambda_{2}\right)=r\left(\lambda_{2}\right) u$, then $T\left(u, \lambda_{1}\right)>T\left(u, \lambda_{2}\right)=r\left(\lambda_{2}\right) u$, implying that $r\left(\lambda_{1}\right)>r\left(\lambda_{2}\right)$. See [23]. It follows that $r:(b, \omega] \rightarrow[0, \infty)$ is strictly decreasing, and it is well known that it is a continuous function of $\lambda$ [26]. Now, we show that by choosing $\omega$ large enough, we can ensure that $r(\omega)<1$. Indeed,

$$
\|T(u, \omega)\|=\left\|K_{\omega}\left(0, \frac{\alpha c}{\omega-b} t(u)\right)\right\| \leq \frac{\alpha c}{\omega-b}\left\|K_{\omega}(0, \cdot)\right\|\|u\|_{\infty},
$$

so, as $\frac{\alpha c}{\omega-b} \rightarrow 0$ as $\omega \rightarrow \infty$, it suffices to show that $\left\|K_{\omega}(0, \cdot)\right\|$ is bounded for large $\omega$. But this is established exactly as in the proof of Proposition 3.4 using the maximum principle (where the notation is $K_{n}(0, f)$ ). As the proof of Proposition 3.4 immediately follows this one, we do not repeat it here. Therefore, $r(\omega) \leq\|T(\bullet, \omega)\|<1$ for large $\omega$. Hereafter, we assume that $\omega$ is large enough that this holds.

For $u \geq 0$ and $b<\lambda<\omega$ we have

$$
T(u, \lambda) \geq K_{\omega}\left(0, \frac{\alpha c}{\lambda-b} t(u)\right)=\frac{\alpha c}{\lambda-b} K_{\omega}(0, t(u)) .
$$

Because $K_{\omega}$ is strongly positive, $K_{\omega}(0, t(1))>0$ (1 stands for the function identically equal to one), so there exists $\delta>0$ such that $K_{\omega}(0, t(1)) \geq \delta 1$. Therefore, $T(1, \lambda) \geq$ $\frac{\alpha \delta c}{\lambda-b} 1$. By Theorem 2.5 [23], $r(\lambda) \geq \frac{\delta \alpha c}{\lambda-b}$, which implies that $r(b+)=\infty$. By the intermediate value theorem, there exists $\lambda^{*}$ satisfying $b<\lambda^{*}<\omega$ such that $r\left(\lambda^{*}\right)=1$.

In order to obtain the desired bounds on $\lambda^{*}$ we argue as follows. By definition of $\lambda^{u}$, there is $\psi>0$ such that $L^{u} \psi=-\lambda^{u} \psi$ and $\psi_{r}=0$ on $\partial_{r} \Omega$ together with the usual boundary conditions at $x=0, L$. Thus, for $r=\lambda^{u}-a+b+c>0$

$$
\begin{aligned}
-L^{u} \psi+(\omega-a) \psi & =\left(\omega-a+\lambda^{u}\right) \psi=(\omega-b-c) \psi+r \psi, \\
d_{r}^{u} \psi_{r}+\alpha \psi & =\frac{\alpha c}{b+c-b} \psi,
\end{aligned}
$$


which implies that

$$
\psi=T(\psi, b+c)+K_{\omega}(r \psi, 0) \geq(1+\delta) T(\psi, b+c)
$$

for small enough $\delta>0$. It follows that $\rho((1+\delta) T(\bullet, b+c))=(1+\delta) r(b+c) \leq 1$, so $r(b+c)<1$, implying that $\lambda^{*}<b+c$. Indeed, if $L$ is a strongly positive and compact linear operator and $L \psi \leq \psi$ for some $\psi>0$, then $L^{n} \psi \rightarrow u \geq 0$. If $u \neq 0$, then, as $u=L u, u>0$ and $\rho(L)=1$ by the Krein-Rutman theorem. If $u=0$, the same theorem implies the existence of $v>0$ such that $L v=\rho(L) v$ and, as $v \leq c \psi$ for some $c>0$, we conclude that $\rho(L)^{n} v \leq c L^{n} \psi$ and hence $\rho(L)^{n} \rightarrow 0$. In either case, we have $\rho(L) \leq 1$.

If $b<a-\lambda^{u}$, then (7.7) can be expressed as

$$
\begin{aligned}
-L^{u} \psi+(\omega-a) \psi & =\left(\omega-a+\lambda^{u}\right) \psi, \\
d_{r}^{u} \psi_{r}+\alpha \psi & =\frac{\alpha c}{a-\lambda^{u}-b} \psi-\frac{\alpha r}{\left(a-\lambda^{u}-b\right)} \psi,
\end{aligned}
$$

which implies that

$$
\psi=T\left(\psi, a-\lambda^{u}\right)-K_{\omega}\left(0, \frac{\alpha r}{\left(a-\lambda^{u}-b\right)} t(\psi)\right)<T\left(\psi, a-\lambda^{u}\right) .
$$

This implies that $r\left(a-\lambda^{u}\right)>1$, so $\lambda^{*}>a-\lambda^{u}$.

In summary, if $b+c>a-\lambda^{u}$, then we have shown the existence of a principal eigenvalue $\lambda^{*}$ satisfying $b<\lambda^{*}<b+c$ and $\lambda^{*}>a-\lambda^{u}$ if also $b<a-\lambda^{u}$.

Now, as $r(\lambda)$ is a simple eigenvalue of $T(\cdot, \lambda)$ (see [23]), the axial symmetry of $u(\lambda)$ follows from an argument identical to the one used when $b+c \leq a-\lambda^{u}$. Similarly, the simplicity of $\lambda^{*}$ as an eigenvalue of (3.10) follows from the simplicity of $r\left(\lambda^{*}\right)$ or it could be argued using results in [2].

Finally, we establish the uniqueness of $\lambda^{*}$ : no other eigenvalue of (3.10) corresponds to a positive eigenvector $(u, w)$. Let $\lambda_{2}>\lambda_{1}$ be two such eigenvalues with eigenvectors $\left(u_{i}, w_{i}\right) \geq 0$. As noted in the beginning of this proof, $\lambda_{i}>b$. Choose $\omega>0$ such that $a+\omega-\lambda_{i}>0, i=1,2$. Then $u_{i}$ satisfies

$$
\begin{aligned}
-L^{u} u+\omega u & =\eta\left[a+\omega-\lambda_{i}\right] u, \\
d_{r}^{u} u+\alpha u & =\eta \alpha \frac{c}{\lambda_{i}-b} u
\end{aligned}
$$

for $\eta=\eta_{i}=1$. As $u_{i}>0$ in $\bar{\Omega}$, it must be the principal eigenvector, corresponding to principal eigenvalue $\eta=\eta_{i}$ in the sense of Theorem 2.2 of [2]. Although Amann assumes a smooth boundary for his domain, Theorem 2.2 [2] holds in our case using arguments like those used in Theorem 6.3. But $a+\omega-\lambda_{1}>a+\omega-\lambda_{2}$ and $\alpha \frac{c}{\lambda_{1}-b}>\alpha \frac{c}{\lambda_{2}-b}$, so by the comparison-of-principal-eigenvalue part of Theorem 2.2 [2] we must have $\eta_{1}>\eta_{2}$. This contradicts that $\eta_{i}=1$ for $i=1,2$. Thus $\lambda_{1}=\lambda_{2}$ as asserted.

Now we prove Proposition 3.4. We begin by showing that $\Re(\lambda) \leq \lambda^{*}$ for all $\lambda$ belonging to the spectrum of the operator $A$ associated with the eigenvalue problem (3.10). Consider the operator $A$, defined on $D(A) \subset Y \equiv C(\bar{\Omega}) \times C\left(\partial_{r} \Omega\right)$. Here

$$
A(u, w)=\left(L^{u} u+a u, b w+\alpha u\right)
$$

for $(u, w) \in D(A)$, the latter defined as

$$
\left\{(u, w) \in\left(C^{2}(\Omega) \cap C^{1+\alpha}(\bar{\Omega})\right) \times C^{\alpha}\left(\partial_{r} \Omega\right): 0=d_{r}^{u} u_{r}+\alpha u-c w\right. \text { and }
$$

Danckwert's BC for $\mathrm{u}$ at $x=0, L\}$. 
Here, $c=f_{w}\left(S^{0}\right)(1-G(0))+\beta$. Actually, $A$ is obtained as a closure of the differential operator above in $Y$ and $D(A)$ is the domain of the closed operator. Given $(g, f) \in Y$ and an increasing sequence of real numbers $\mu_{n} \rightarrow \infty$, consider the equations $\left(\mu_{n}-\right.$ $A)(u, w)=(g, f)$. This system becomes (we assume Danckwerts' boundary conditions at $x=0, L)$

$$
\begin{aligned}
-L^{u} u+\left(\mu_{n}-a\right) u & =g, \\
d_{r}^{u} u_{r}+\alpha u & =c w, \\
\left(\mu_{n}-b\right) w-\alpha u & =f,
\end{aligned}
$$

which is equivalent to

$$
\begin{aligned}
u & =K_{n}(g, 0)+c K_{n}(0, w), \\
w & =\left(\mu_{n}-b\right)^{-1} f+\alpha\left(\mu_{n}-b\right)^{-1} t(u),
\end{aligned}
$$

where $t$ is the restriction (to $\partial_{r} \Omega$ ) operator and $K_{n}$ is the inverse operator associated with $-L^{u}+\left(\mu_{n}-a\right)$ (see Theorem 6.1). If we define the positive bounded operators

$$
\begin{aligned}
T_{0}^{n}(u, w) & =\left(c K_{n}(0, w), \alpha\left(\mu_{n}-b\right)^{-1} t(u)\right), \\
T_{1}^{n}(g, f) & =\left(K_{n}(g, 0),\left(\mu_{n}-b\right)^{-1} f\right)
\end{aligned}
$$

on $Y$, then the pair of equations takes the form

$$
\left(I-T_{0}^{n}\right)(u, w)=T_{1}^{n}(g, f) .
$$

We will show that for large $n$, the spectral radius of $T_{0}^{n}$ is strictly less than unity, which implies the solvability of the operator equation

$$
(u, w)=\left(\mu_{n}-A\right)^{-1}(g, f)=\left(I-T_{0}^{n}\right)^{-1} T_{1}^{n}(g, f)=\sum_{k \geq 0}\left(T_{0}^{n}\right)^{k} T_{1}^{n}(g, f)
$$

as well as the positivity of the resolvent operator (recall $T_{i}^{n} \geq 0$ for $\left.i=0,1\right):\left(\mu_{n}-\right.$ $A)^{-1} \geq 0$ for large $n$.

Now $v_{n}=K_{n}(0, f)$ satisfies

$$
\begin{aligned}
-L^{u} u+\left(\mu_{n}-a\right) u & =0, \\
d_{r}^{u} u_{r}+\alpha u & =f,
\end{aligned}
$$

so $U=v_{n}-v_{m}, m>n$, satisfies

$$
\begin{aligned}
-L^{u} U+\left(\mu_{n}-a\right) U & =\left(\mu_{m}-\mu_{n}\right) v_{m} \\
d_{r}^{u} u_{r}+\alpha u & =0 .
\end{aligned}
$$

If $f \geq 0$, then so is $v_{n} \geq 0$ for all large $n$ and, consequently, $U=\left(K_{n}-K_{m}\right)(0, f) \geq 0$ when $m>n$. For arbitrary $f=f^{+}-f^{-}$, this leads immediately to

$$
-K_{n}(0,|f|) \leq-K_{n}\left(0, f^{-}\right) \leq K_{m}(0, f) \leq K_{n}\left(0, f^{+}\right) \leq K_{n}(0,|f|),
$$

so we conclude that $M=\sup _{n \geq 0}\left\|K_{n}(0, \cdot)\right\|<\infty$. A calculation gives

$$
\left(T_{0}^{n}\right)^{2}(u, w)=c \alpha\left(\mu_{n}-b\right)^{-1}\left(K_{n}(0, t(u)), t\left(K_{n}(0, w)\right)\right),
$$


which implies that $\left\|T_{0}^{n}\right\|^{2} \leq c \alpha\left(\mu_{n}-b\right)^{-1} M<1$ for large $n$ and shows that the spectral radius $r\left(T_{0}^{n}\right)<1$ as claimed. It follows from Theorem 1.1 of [25] that if $\mu_{0}=\inf \left\{\mu \in R:(\mu-A)^{-1} \in L(Y),(\mu-A)^{-1} \geq 0\right\}$, then $\mu_{0}$ belongs to the spectrum of $A$ if it exceeds $-\infty$ and if $\Re(\lambda)>\mu_{0}$, then $\lambda$ belongs to the resolvent set of $A$ and, finally, $\mu>\mu_{0}$ implies that the spectral radius $r\left((\mu-A)^{-1}\right)=\left(\mu-\mu_{0}\right)^{-1}$. As $\lambda^{*}$ belongs to the spectrum of $A, \mu_{0}$ is finite. We will apply Theorem 1.2 of [25] to show that $\mu_{0}=\lambda^{*}$.

It is easy to see that $\left(T_{0}^{n}\right)^{2}$ and $T_{0}^{n} T_{1}^{n}$ are compact operators so

$$
\left(\mu_{n}-A\right)^{-1}=\sum_{k \geq 0}\left(T_{0}^{n}\right)^{k} T_{1}^{n}=T_{1}^{n}+\text { compact operator. }
$$

It follows [25] that the essential spectral radius $r_{e}\left(\left(\mu_{n}-A\right)^{-1}\right)=r_{e}\left(T_{1}^{n}\right)$. As $T_{1}^{n}$ is a direct sum of two operators, its spectrum is easily resolved. It consists of the union of $\left(\mu_{n}-b\right)^{-1}$ and the spectrum of the compact operator $K_{n}(\cdot, 0) .\left(\mu_{n}-b\right)^{-1}$ is an eigenvalue of infinite multiplicity so it belongs to the essential spectrum of $T_{1}^{n}$ while the spectrum of $K_{n}(\cdot, 0)$, excepting zero, are eigenvalues of finite multiplicity. Hence, we conclude that $r_{e}\left(T_{1}^{n}\right)=\left(\mu_{n}-b\right)^{-1}$ so $r_{e}\left(\left(\mu_{n}-A\right)^{-1}\right)=\left(\mu_{n}-b\right)^{-1}$. If $r_{e}\left(\left(\mu_{n}-A\right)^{-1}\right)=$ $r\left(\left(\mu_{n}-A\right)^{-1}\right)$, then it follows from the above that $\left(\mu_{n}-b\right)^{-1}=\left(\mu_{n}-\mu_{0}\right)^{-1}$, so $b=\mu_{0}$. But this contradicts that $\lambda^{*}>b$ since Theorem 1.1 of [25] implies that $\lambda^{*}$ would belong to the resolvent set of $A$. Thus $r_{e}\left(\left(\mu_{n}-A\right)^{-1}\right)<r\left(\left(\mu_{n}-A\right)^{-1}\right)$, so Theorem 1.2 and Remark 1.3 of [25] apply. The latter asserts the existence of a positive eigenvector for $A$ corresponding to the eigenvalue $\mu_{0}$. But $\lambda^{*}$ is the unique eigenvalue with a positive eigenvector. Therefore, $\mu_{0}=\lambda^{*}$ and $\lambda$ belongs to the resolvent set of $A$ whenever $\Re(\lambda)>\lambda^{*}$.

Now we return to consider operator $\mathcal{L}$, defined on $D(\mathcal{L}) \subset X$. Given $(h, g, f) \in X$ consider the equation $(\lambda-\mathcal{L})(S, u, w)=(h, g, f)$ for $(S, u, w) \in D(\mathcal{L})$. It has a solution if and only if $(u, w) \in D(A),(\lambda-A)(u, w)=(g, f)$, and $S$ satisfies

$$
\begin{aligned}
-L^{S} S+\lambda S & =\gamma^{-1} u f_{u}\left(S^{0}\right)+h, \\
d_{r}^{S} S_{r} & =-\gamma^{-1} w f_{w}\left(S^{0}\right) .
\end{aligned}
$$

If $\Re(\lambda)>\lambda^{*}$, then $(u, w)=(\lambda-A)^{-1}(g, f)$, and if $\lambda$ does not belong to the spectrum of the operator associated with eigenvalue problem (3.9), then $S$ may be solved in terms of the right-hand side of this equation. As $-\lambda^{S}$ is the principal eigenvalue of (3.9), it follows that this last equation may be solved whenever $\Re(\lambda)>-\lambda^{S}$. Therefore, if $\Re(\lambda)>\max \left\{-\lambda^{S}, \lambda^{*}\right\}$, then $\lambda$ belongs to the resolvent set of $\mathcal{L}$.

8. Appendix. Existence of positive steady state. We prove Theorem 3.5.

Proof of Theorem 3.5. The proof follows closely the proof of Theorem 4.1 in [5] and in particular we will use Lemma A.2 there, which concerns the solution of the algebraic third equation of (3.11). There, it is shown (with $S^{0}$ scaled to unity and a parameter $\epsilon$ which here becomes unity) that the third equation can be solved for a smooth function $w=h(S, u) \in\left(0, w_{\infty}\right)$ when $u>0$ and $0 \leq S \leq S^{0}$. The behavior of this solution as $u \rightarrow 0$ depends on the sign of $b$, tending to 0 for $b<0$ and tending to a nontrivial function $w_{*}(S)$ as $u \rightarrow 0$ when $b>0$. It is convenient to make the change of variables $v=S^{0}-S$ so that $v$ satisfies homogeneous boundary conditions at $x=0$. Define

$$
H_{1}(S, u, w)=\alpha u W+w\left[f_{w}(S)(1-G(W))+\beta\right]
$$


and observe that $H_{1} \geq 0$ is monotone increasing in all its arguments. With this notation, we may write the radial boundary condition for $u$ as

$$
d_{r}^{u} u_{r}+\alpha u=H_{1}(S, u, w) .
$$

We then have the system

$$
\begin{aligned}
-L^{S} v & =\gamma^{-1} u f_{u}\left(S^{0}-v\right), \\
-L^{u} u+\omega u & =u\left[f_{u}\left(S^{0}-v\right)-k+\omega\right], \\
w & =h\left(S^{0}-v, u\right),
\end{aligned}
$$

where $\omega>0$ is chosen such that $f_{u}\left(S^{0}\right)-k+\omega>0$, with radial boundary conditions

$$
\begin{aligned}
d_{r}^{S} v_{r} & =\gamma^{-1} w f_{w}\left(S^{0}-v\right), \\
d_{r}^{u} u_{r}+\alpha u & =H_{1}\left(S^{0}-v, u, w\right) .
\end{aligned}
$$

We will always interpret $S^{0}-v$ as the positive part of it- $\left(S^{0}-v\right)_{+}$.

Convert to a fixed point problem. We can invert the differential operators on the left to express the boundary value problem as a fixed point problem (see Theorem 6.2):

$$
\begin{aligned}
v & =T_{1}(v, u) \equiv S_{1}\left(\gamma^{-1} u f_{u}\left(S^{0}-v\right), \gamma^{-1} w f_{w}\left(S^{0}-v\right)\right), \\
u & =T_{2}(v, u) \equiv S_{\omega}\left(u\left[f_{u}\left(S^{0}-v\right)-k+\omega\right], H_{1}\left(S^{0}-v, u, w\right)\right), \\
w & =h\left(S^{0}-v, u\right) .
\end{aligned}
$$

We view (8.4) as the fixed point equation

$$
(v, u)=T(v, u)
$$

where $T: Y_{+} \rightarrow Y_{+}$is defined by the right side of (8.4) and $Y_{+}=C_{+}(\bar{\Omega}) \times C_{+}(\bar{\Omega})$ is the positive cone. Obviously, $T(0,0)=(0,0)$, corresponding to the washout steady state, but we seek a nontrivial fixed point. It follows from well-known arguments (see Theorem 6.1) that $T$ is a completely continuous mapping on $Y_{+}$.

Obtain a priori bounds. First we show that if $T(v, u)=\eta(v, u)$ for some $(v, u) \in Y_{+}$and $\eta \geq 1$, then $v \leq S^{0}$ in $\Omega$. Indeed, $v$ satisfies the appropriate equation from (8.2) with boundary conditions from (8.3) except that $\gamma^{-1}$ is replaced by $(\eta \gamma)^{-1}$. It is convenient to show instead that $S \geq 0 . S$ satisfies

$$
\begin{aligned}
-L^{S} S & =-(\gamma \eta)^{-1} u f_{u}\left(S_{+}\right), \\
d_{r}^{S} S_{r} & =-(\gamma \eta)^{-1} w f_{w}\left(S_{+}\right), \\
v(r) S^{0} & =-d_{x}^{S} S_{x}+v(r) S, \quad x=0, \\
0 & =S_{x}, \quad x=L,
\end{aligned}
$$

where $\left(S^{0}-v\right)_{+}=S_{+}$, the positive part of $S$. We use $S=S_{+}+S_{-}$and the fact that

$$
f_{u}\left(S_{+}\right) S_{-}=f_{w}\left(S_{+}\right) S_{-}=0 .
$$

Multiply the equation by $S_{-}$and integrate over $\Omega$ to get $\left(D=\operatorname{diag}\left(d_{x}^{S}, d_{r}^{S}, d_{r}^{S}\right)\right)$ :

$$
0=\int_{\Omega} D \nabla\left(S_{-}\right) \cdot \nabla\left(S_{-}\right) d V+\int_{x=0} v(r)\left(S-S^{0}\right) S_{-} d A+\int_{\Omega} v(r) S_{x} S_{-} d V
$$




$$
\begin{gathered}
=\int_{\Omega} D \nabla\left(S_{-}\right) \cdot \nabla\left(S_{-}\right) d V+\int_{x=0} v(r)\left(S-S^{0}\right) S_{-} d A \\
\quad+\int_{x=L} v(r) \frac{\left(S_{-}\right)^{2}}{2} d A-\int_{x=0} v(r) \frac{\left(S_{-}\right)^{2}}{2} d A \\
=\int_{\Omega} D \nabla\left(S_{-}\right) \cdot \nabla\left(S_{-}\right) d V+\int_{x=0} v(r) S^{0}\left(-S_{-}\right) d A \\
\quad+\int_{x=L} v(r) \frac{\left(S_{-}\right)^{2}}{2} d A+\int_{x=0} v(r) \frac{\left(S_{-}\right)^{2}}{2} d A .
\end{gathered}
$$

Noting that every term on the right side is nonnegative, we find that $\nabla\left(S_{-}\right)=0$ a.e. so $S_{-}$is constant. The only constant consistent with $S$ satisfying the equation above is $S_{-} \equiv 0$. Thus, $S \geq 0$, so $v \leq S^{0}$.

We now show that there exists $M>0$ such that $T(v, u)=\eta(v, u)$ has no solution $(v, u) \in Y_{+}$with $\|v\|+\|u\|=M$ and $\eta \geq 1$. Observe that $u$ satisfies

$$
\begin{aligned}
-L^{u} u+\omega u & =\eta^{-1} u\left[f_{u}\left(S^{0}-v\right)-k+\omega\right], \\
d_{r}^{u} u_{r}+\alpha\left[1-\eta^{-1} W\right] u & =\eta^{-1} w\left[f_{w}\left(S^{0}-v\right)(1-G(W))+\beta\right]
\end{aligned}
$$

for $\eta \geq 1$ and, of course, homogeneous Danckwert's boundary conditions at $x=0$ and $x=L$. For simplicity, we let $\chi(x, y, z) \equiv \alpha\left[1-\eta^{-1} W(x, y, z)\right], f(x, y, z) \equiv$ $\eta^{-1} w\left[f_{w}\left(S^{0}-v\right)(1-G(W))+\beta\right]$, and $c(x, y, z) \equiv \eta^{-1}\left[f_{u}\left(S^{0}-v\right)-k+\omega\right]$, and note that they are bounded (uniformly in $\eta \geq 1$ ) and nonnegative. In particular, there exists $F>0$ such that $\|f\| \leq F$ for all $\eta \geq 1$.

Recall that (see (3.1)) we have an a priori $L^{1}$ bound for $u$ :

$$
\int_{\Omega} u d V \leq B
$$

which is independent of $\eta \geq 1$ since $\eta^{-1} f_{u} \leq f_{u}$ and $\eta^{-1} f_{w} \leq f_{w}$ for $\eta \geq 1$. Multiply the equation by $u$ and integrate to get

$$
\int_{\Omega} d_{x}^{u} u_{x}^{2}+d_{r}^{u}\left(u_{y}^{2}+u_{z}^{2}\right)+v(r) u_{x} u+\omega u^{2} d V=\int_{\Omega} c u^{2} d V-\int_{x=0} v(r) u^{2} d A+\int_{\partial_{r} \Omega} u(f-\chi u) d A .
$$

Using

$$
\int_{\Omega} v(r) u_{x} u d V=\int_{x=L} v(r) \frac{u^{2}}{2} d A-\int_{x=0} v(r) \frac{u^{2}}{2} d A
$$

we find that

$$
\int_{\Omega} d_{x}^{u} u_{x}^{2}+d_{r}^{u}\left(u_{y}^{2}+u_{z}^{2}\right)+\omega u^{2} d V \leq \int_{\Omega} c u^{2} d V+\int_{\partial_{r} \Omega} u f d A
$$

Now, using inequalities such as

$$
\begin{aligned}
\int_{\Omega} c u^{2} d V & \leq \epsilon \int_{\Omega}\left[|D u|^{2}+u^{2}\right] d V+C(\epsilon)\left(\int_{\Omega} u d V\right)^{2} \\
\int_{\partial_{r} \Omega}|f u| d A & \leq \epsilon \int_{\partial_{r} \Omega}|u|^{2} d A+C(\epsilon) F^{2} \\
& \leq \epsilon\left[\int_{\Omega}\left[|D u|^{2}+u^{2}\right] d V+C\left(\int_{\Omega} u d V\right)^{2}\right]+C(\epsilon) F^{2}
\end{aligned}
$$


where $C, C(\epsilon)$ are independent of $\eta \geq 1$ (the first inequality follows from Young's inequality and the Gagliardo-Nirenberg inequality as on p. 138 of [33]), and choosing $\epsilon>0$ suitably small, there are $d, \delta>0, C$ independent of $\eta \geq 1$ such that

$$
\delta \int_{\Omega} u^{2} d V \leq \int_{\Omega} d|D u|^{2}+\delta u^{2} d V \leq C\left[F^{2}+\left(\int_{\Omega} u d V\right)^{2}\right] .
$$

Hence, we have an a priori bound for the $L^{2}$ norm of $u$, independent of $\eta \geq 1$ :

$$
\delta \int_{\Omega} u^{2} d V \leq \int_{\Omega} d|D u|^{2}+\delta u^{2} d V \leq C\left[F^{2}+B^{2}\right] .
$$

Now, we may apply the Moser iteration scheme as in Theorem 8.15 of [21] to obtain a uniform $L^{\infty}$ estimate of $u$, independent of $\eta \geq 0$. As $v \leq S^{0}$, we have established the required bound.

Application of a fixed point theorem in case $b=f_{w}\left(S^{0}\right) G(0)-k_{w}-$ $\boldsymbol{\beta}<\mathbf{0}$. The remainder of the proof breaks down into two cases. We first suppose that $b=f_{w}\left(S^{0}\right) G(0)-k_{w}-\beta<0$. In this case, we use Theorem 1.6 in [32] (see also Theorem 13.2 in [1]). A computation and use of Lemma A.2 in [5] shows that $T$ has a right derivative $T_{+}^{\prime}(0)$ and that

$$
\begin{aligned}
T_{+}^{\prime}(0)(V, U)= & \left(S_{1}\left(\gamma^{-1} U f\left(S^{0}\right), \gamma^{-1} f_{w}\left(S^{0}\right) \frac{\alpha}{-b} U\right), S_{\omega}\left(U\left[f\left(S^{0}\right)-k+\omega\right],\right.\right. \\
& \left.\left.\frac{\alpha\left[f_{w}\left(S^{0}\right)(1-G(0))+\beta\right]}{-b} U\right)\right) .
\end{aligned}
$$

If $\lambda(V, U)=T_{+}^{\prime}(0)(V, U)$ with $(V, U)>(0,0)$ and $\lambda>0$, then we have

$$
\begin{aligned}
-L^{S} V & =\lambda^{-1} \gamma^{-1} U f_{u}\left(S^{0}\right), \\
-L^{u} U+\omega U & =\lambda^{-1} U\left[f_{u}\left(S^{0}\right)-k+\omega\right]
\end{aligned}
$$

with radial boundary conditions

$$
\begin{aligned}
d_{r}^{S} V_{r} & =\lambda^{-1} \gamma^{-1} f_{w}\left(S^{0}\right) \frac{\alpha}{-b} U, \\
d_{r}^{u} U_{r}+\alpha U & =\lambda^{-1} \frac{\alpha c}{-b} U,
\end{aligned}
$$

where $c=f_{w}\left(S^{0}\right)(1-G(0))+\beta$. Note the appearance of $\lambda$ in the boundary conditions. Observe that for each $\lambda>0$ and $U>0$, there is a unique $V$ satisfying the equations and boundary conditions above. Furthermore, the equation and boundary conditions for $U$ are independent of $V$ and, therefore, may be considered separately. This equation for $U$ is just the kind of eigenvalue problem treated by Amann in [2] where the existence of a principal eigenvalue, unique among eigenvalues in that it is the only one with corresponding positive eigenvector, is established (see Theorem 2.2 [2]) as well as a comparison principle which we will find useful. Although Amann assumes a smooth boundary for his domain, Theorem 2.2 [2] holds in our case using arguments like those used in Theorem 6.3. Recall that the eigenvalue $\lambda^{*}$ and the second component of its corresponding eigenvector $(\bar{S}, \bar{u}, \bar{w})$ satisfy

$$
\begin{aligned}
-L^{u} \bar{u}+\omega \bar{u} & =\eta\left[f_{u}\left(S^{0}\right)-k+\omega-\lambda^{*}\right] \bar{u}, \\
d_{r}^{u} \bar{u}+\alpha \bar{u} & =\eta \alpha \frac{c}{\lambda^{*}-b} \bar{u}
\end{aligned}
$$


with $\eta=1$ and where $\eta$ is displayed for comparison with $\lambda^{-1}$ appearing in (8.7)(8.8). Since $\bar{u}>0, \eta=1$ is the principal eigenvalue of (8.9) in the sense of [2]. As $f_{u}\left(S^{0}\right)-k+\omega-\lambda^{*}<f_{u}\left(S^{0}\right)-k+\omega$ and $\alpha \frac{c}{\lambda^{*}-b}<\alpha \frac{c}{-b}$, Theorem 2.2 of [2] implies that the principal eigenvalue $\lambda^{-1}$ for (8.7)-(8.8) must be smaller than one, the principal eigenvalue of (8.9). Thus, $\lambda>1$ and, again by the uniqueness of the principal eigenvalue, one is not an eigenvalue of (8.7) with a corresponding positive eigenvector. It follows from Theorem 13.2 of [1] or Theorem 1.6 in [32] that $T$ has a fixed point $(v, u) \in Y_{+}$with $0<\|u\|+\|v\|<M$.

Application of a fixed point theorem in case $b=f_{w}\left(S^{0}\right) G(0)-k_{w}-$ $\boldsymbol{\beta}>\mathbf{0}$. Now suppose that $b=f_{w}(1) G(0)-k_{w}-\beta>0$ holds. In this case, by Lemma A.2 of [5], there exists $S^{*}<S^{0}$ such that $w=h(S, u) \geq w_{*}(S)$ and $h(S, u) \rightarrow w_{*}(S)$ as $u \rightarrow 0$, where $w_{*}(S)>0$ for $S^{*}<S \leq S^{0}$ and vanishes on $\left[0, S^{*}\right]$. We will use Theorem 12.3 in [1], which requires us to find $0<\delta<M$ such that $T(v, u) \leq(v, u)$ does not hold for any $(v, u) \in Y_{+}$with $\|v\|+\|u\|=\delta$. (Actually, we could also use Corollary 12.4 in [1].) Suppose, for the sake of contradiction, that such a $(v, u)$ exists. Then $0 \leq v \leq \delta$, and from the definition of $T$ and monotonicity properties of the various functions, we have

$$
\begin{aligned}
v & \geq S_{1}\left(\gamma^{-1} u f_{u}\left(S^{0}-v\right), \gamma^{-1} w f_{w}\left(S^{0}-v\right)\right) \\
& \geq S_{1}\left(0, \gamma^{-1} w f_{w}\left(S^{0}-v\right)\right) \\
& \geq \gamma^{-1} f_{w}\left(S^{0}-\delta\right) w_{*}\left(S^{0}-\delta\right) S_{1}(0,1) \\
& \geq \gamma^{-1} f_{w}\left(S^{0}-\delta\right) w_{*}\left(S^{0}-\delta\right) C
\end{aligned}
$$

where $C=\min S_{1}(0,1)$. Recall that $w=h(S, u)>w_{*}(S)$ and that $w_{*}(S)$ is strictly increasing in $S$ for $S>S^{*}$ by Lemma A.2 in [5]. Thus we have

$$
\delta \geq C f_{w}\left(S^{0}-\delta\right) w_{*}\left(S^{0}-\delta\right) .
$$

Choosing $\delta$ small enough we have a contradiction as $f_{w}\left(S^{0}\right), w_{*}\left(S^{0}\right)>0$. Thus, by Theorem 12.3 of [1], $T$ has a fixed point with $\delta<\|v\|+\|u\|<M$.

Positivity of $\boldsymbol{u}$ and $\boldsymbol{w}$. Theorem 4 of [15] may be applied to (8.2) and (8.3) to conclude that either $u \equiv 0$ or $u(x, y, z)>0$ in $\bar{\Omega}$. But from the equation for $u$ and the definition of $H_{1}$, if $u \equiv 0$, then we must have $w \equiv 0$ and hence $v \equiv 0$, a contradiction. We conclude that $u$ must be positive, so $w=h\left(S^{0}-v, u\right)>0$ as well.

Symmetry of solutions. We do not attempt to prove that the solutions that we have produced thus far are symmetric. Rather, we observe that we can set up our fixed point problem (8.4) in the closed subspace $Y^{s}$ of $Y$ consisting of radially symmetric functions. By virtue of the last assertion of Theorem 6.1, the operator $T: Y_{+}^{s} \rightarrow Y_{+}^{s}$. As a symmetric solution in $Y_{+}^{s}$ is also in $Y_{+}$, our a priori bound argument and other estimates apply to symmetric solutions as well. Therefore the same argument as above leads to a symmetric solution.

9. Appendix. Existence of solutions for the parabolic problem. This section contains a proof of Theorem 2.1. We begin with an existence result taken from Theorem 5.1, Chap. 3 in [22]. The regularity assertions follow from Corollary 4.2 of [16]. Fix $T>0$ and let $D_{T} \equiv\{(x, y, z, t) \in \Omega \times(0, T)\}$.

LEMma 9.1 (existence for the linear equation). Let $f \in L^{\infty}\left(D_{T}\right), g \in L^{\infty}\left(S_{T}\right)$, and $S_{0} \in C(\Omega)$. Then the $I B V P$

$$
\begin{aligned}
S_{t}-L^{S} S+c S & =g(x, y, z, t) \quad \text { in } \Omega, \\
d_{r}^{S} S_{r}+b S & =f(x, y, z, t), \quad r=R, \\
S(x, y, z, 0) & =S_{0}(x, y, z),
\end{aligned}
$$


together with the boundary conditions (2.4) and (2.5), has a unique weak solution $S \in V_{2}\left(D_{T}\right)$ (see [22]) which is Hölder continuous in $(x, y, z, t)$ uniformly in $\bar{\Omega} \times\left[T^{\prime}, T\right]$ for each $0<T^{\prime}$. In fact, the Hölder exponent and Hölder norm of $S$ depend only on the $L^{\infty}$ norm of $S$ on $\bar{\Omega} \times\left[T^{\prime}, T\right]$.

By a weak solution of (9.1) we mean that $S$ satisfies

$$
\begin{gathered}
-\iint_{D_{T}} S \eta_{t} d V d t+\iint_{D_{T}}\left[d_{x}^{S} S_{x} \eta_{x}+d_{r}^{S}\left(S_{y} \eta_{y}+S_{z} \eta_{z}\right)+v(r) S_{x} \eta+c S \eta\right] d V d t \\
=\iint_{D_{T}} g \eta d V d t+\int_{0}^{T} \int_{x=0} v(r)\left(S^{0}-S\right) d A d t \\
\quad+\int_{0}^{T} \int_{\partial_{r} \Omega} \eta(f-b S) d A d t+\int_{\Omega} S_{0} \eta(x, y, z, 0) d V
\end{gathered}
$$

for all $\eta \in W_{2}^{1,1}\left(D_{T}\right)$ for which $\eta(x, y, z, T)=0$ a.e. in $\Omega$. See Chapter 3, sect. 5 in [22]. The following result, adapted from [15], concerns a function $S$ satisfying

$$
\begin{aligned}
& S_{t}-L^{S} S+c S \geq 0 \quad \text { in } \Omega, \\
& d_{r}^{S} S_{r}+b S \geq 0, \quad r=R,
\end{aligned}
$$

and the boundary conditions (2.4) and (2.5) in a weak sense.

Lemma 9.2 (positivity lemma). Let $S \in V_{2}\left(D_{T}\right)$ satisfy (9.2) in the weak sense (see [15]). If $S(x, y, z, 0) \geq 0, b \geq 0$, and $c$ is bounded on $D_{T}$, then $S \geq 0$ a.e. in $\overline{D_{T}}$.

Let us now construct upper and lower solutions for our system. In order to do this without repeating certain boundary and initial conditions over and over again, let us agree that, hereafter, all equations for an $S(u)$ will be assumed to satisfy the same initial conditions (2.7) and the same boundary conditions (2.4) and (2.5). The equation itself and the radial boundary conditions may vary, but not these conditions. Similarly, all equations for $w$ will satisfy the initial conditions for $w$ as above. This convention will free us from restating these conditions. A solution is always to be interpreted as a weak solution according to Lemma 9.1.

It will be convenient to define

$$
H_{1}(S, u, w)=\alpha u W+w\left[f_{w}(S)(1-G(W))+\beta\right]
$$

and

$$
H_{2}(S, u, w)=w f_{w}(S) g(W)+\alpha u
$$

and observe that according to our assumptions, $H_{i} \geq 0$ is monotone increasing in all its arguments. With this notation, we may write the radial boundary condition for $u$ as

$$
d_{r}^{u} u_{r}+\alpha u=H_{1}(S, u, w)
$$

and the equation for $w$ as

$$
w_{t}+\left(k_{w}+\beta\right) w=(1-W) H_{2}(S, u, w) .
$$

Let $Y=(S, u, w)$ below. 
Upper and lower solutions are trivial for (2.3):

$$
\hat{w} \equiv 0, \quad \tilde{w} \equiv w_{\infty}
$$

As for the lower solutions for (2.1), we set

$$
\hat{S} \equiv 0, \quad \hat{u} \equiv 0 .
$$

The upper solution for $S$, denoted by $\tilde{S}$, satisfies

$$
S_{t}=L^{S} S
$$

and all initial and boundary conditions as above except for

$$
d_{r}^{S} S_{r}=0
$$

replace the radial boundary condition. Observe that this is a linear equation so there exists a unique globally defined weak solution $\tilde{S}$ satisfying $\tilde{S} \geq 0$ by the positivity lemma. In fact,

$$
\tilde{S} \rightarrow S^{0}, \quad t \rightarrow \infty
$$

uniformly in $(x, y, z) \in \Omega$. Furthermore, if $(S, u, w)$ is a solution of our system satisfying the range conditions, then $S \leq \tilde{S}$ in $\overline{D_{T}}$. Setting $\phi=\tilde{S}-S$, we have

$$
\begin{aligned}
\phi_{t}-L^{S} \phi & =\gamma^{-1} u f_{u}(S) \geq 0, \\
d_{r}^{S} \phi_{r} & =\gamma^{-1} w f_{w}(S) \geq 0,
\end{aligned}
$$

and $\phi$ satisfies homogeneous initial and boundary conditions except as described above. By the positivity lemma, $\phi \geq 0$, proving our assertion.

Define the upper solution $\tilde{u}$ as the solution of

$$
\begin{aligned}
u_{t} & =L^{u} u+u\left[f_{u}(\tilde{S})-k\right], \\
d_{r}^{u} u_{r} & =w_{\infty}\left[f_{w}(\tilde{S})+\beta\right],
\end{aligned}
$$

with all initial and boundary conditions except as described above. As this is a linear equation the solution exists globally in time. The positivity lemma implies $\tilde{u} \geq 0$. Furthermore, if $(S, u, w)$ is any solution of our problem satisfying the range conditions, then $u \leq \tilde{u}$ in $\overline{D_{T}}$. Indeed, set $\psi=\tilde{u}-u$ and observe that it satisfies

$$
\begin{aligned}
\psi_{t}-L^{u} \psi-\psi\left[f_{u}(\tilde{S})-k\right] & =u\left[f_{u}(\tilde{S})-f_{u}(S)\right] \geq 0 \\
d_{r}^{u} \psi_{r}=w_{\infty}[ & \left.f_{w}(\tilde{S})+\beta\right]+\alpha u(1-W) \\
& \quad-w\left[f_{w}(S)(1-G(W))+\beta\right] \geq \alpha u(1-W) \geq 0
\end{aligned}
$$

as well as homogeneous initial and boundary conditions. By the positivity lemma, $\psi \geq 0$, proving our claim.

We now begin the construction of a sequence of approximate solutions (following [27], Chapter 9, sect. 9.2, mixed monotone case) satisfying

$$
\begin{gathered}
0=\hat{S} \leq \underline{S}^{1} \leq \underline{S}^{2} \leq \cdots \leq \underline{S}^{n} \leq \bar{S}^{n} \leq \bar{S}^{n-1} \leq \cdots \leq \bar{S}^{1} \leq \tilde{S} \\
0=\hat{u} \leq \underline{u}^{1} \leq \underline{u}^{2} \leq \cdots \leq \underline{u}^{n} \leq \bar{u}^{n} \leq \bar{u}^{n-1} \leq \cdots \leq \bar{u}^{1} \leq \tilde{u}
\end{gathered}
$$




$$
0=\hat{w} \leq \underline{w}^{1} \leq \underline{w}^{2} \leq \cdots \leq \underline{w}^{n} \leq \bar{w}^{n} \leq \bar{w}^{n-1} \leq \cdots \leq \bar{w}^{1} \leq \tilde{w}=w_{\infty} .
$$

For this we first choose $m>0$ such that for all $S \geq 0$

$$
\begin{aligned}
m S-\gamma^{-1} f_{w}(S) & \geq 0, \\
m-\gamma^{-1} f_{w}^{\prime}(S) & \geq 0, \\
m S-\gamma^{-1} \tilde{u} f_{u}(S) & \geq 0, \\
m-\gamma^{-1} \tilde{u} f_{u}^{\prime}(S) & \geq 0,
\end{aligned}
$$

and for all $(x, y, z, t) \in \overline{D_{T}}$. The compactness of $\overline{D_{T}}$ and our conditions on $f_{u}, f_{w}$ ensure that we can find such an $m$.

Let $\bar{S}^{1}$ satisfy

$$
\begin{aligned}
S_{t}-L^{S} S+m S & =m \tilde{S}-\gamma^{-1} \hat{u} f_{u}(\tilde{S})=m \tilde{S} \\
d_{r}^{S} S_{r}+m S & =m \tilde{S}-\gamma^{-1} \hat{w} f_{w}(\tilde{S})=m \tilde{S},
\end{aligned}
$$

together with the other initial and boundary conditions for $S$. Let $\bar{u}^{1}$ satisfy

$$
\begin{aligned}
u_{t} & =L^{u} u+u\left[f_{u}(\tilde{S})-k\right], \\
d_{r}^{u} u_{r}+\alpha u & =H_{1}(\tilde{S}, \tilde{u}, \tilde{w}),
\end{aligned}
$$

together with the other initial and boundary conditions for $u$, and let $\bar{w}^{1}$ satisfy

$$
w_{t}+\left(k_{w}+\beta\right) w=(1-W) H_{2}(\tilde{S}, \tilde{u}, \tilde{w}),
$$

together with initial data for $w$.

In order to estimate solutions of the $w$ equation it's useful to have the following result.

Lemma 9.3 (monotonicity lemma). Let $a(t) \geq 0$. The solution of

$$
v^{\prime}+c v=\left(1-\frac{v}{w_{\infty}}\right) a(t), \quad v(0)=v_{0} \in\left[0, w_{\infty}\right]
$$

satisfies $0 \leq v(t) \leq w_{\infty}$ for $t \geq 0$. Moreover, if $0 \leq a_{1}(t) \leq a_{2}(t)$ and if $v_{i}$ satisfies the ODE with $a=a_{i}$, then $v_{1}(t) \leq v_{2}(t)$ for $t \geq 0$.

Proof. The first assertion is trivial; the second follows since $u=v_{2}-v_{1}$ satisfies $u^{\prime}+\left(c+a_{1} / w_{\infty}\right) u=\left(a_{2}-a_{1}\right)\left(1-v_{2} / w_{\infty}\right) \geq 0, u(0)=0$.

It's easy to see that $\hat{w}=0 \leq \bar{w}^{1} \leq \tilde{w}=w_{\infty}$ from the monotonicity lemma. Also, the positivity lemma implies that $\bar{S}^{1}, \bar{u}^{1} \geq 0$, the latter since $H_{1} \geq 0$. We now verify that $\bar{v}^{1} \leq \tilde{v}$ for $v=S, u, w$.

$\phi=\tilde{S}-\bar{S}^{1}$ satisfies

$$
\begin{aligned}
\phi_{t}-L^{S} \phi+m \phi & =0, \\
d_{r}^{S} \phi+m \phi & =0,
\end{aligned}
$$

together with homogeneous initial and boundary conditions for $S$, so we conclude that $\phi=0$ and $\tilde{S}=\bar{S}^{1}$.

$\psi=\tilde{u}-\bar{u}^{1}$ satisfies

$$
\begin{aligned}
\psi_{t}-L^{u} \psi-\left[f_{u}(\tilde{S})-k\right] \psi & =0, \\
d_{r}^{u} \psi+\alpha \psi & =w_{\infty}\left[f_{w}(\tilde{S})+\beta\right]+\alpha \tilde{u}-\alpha \tilde{u} \tilde{W} \\
-\tilde{w}\left[f_{w}(\tilde{S})(1-G(\tilde{W}))+\beta\right]=0, &
\end{aligned}
$$


(since $\tilde{W}=1$ ) together with homogeneous initial and boundary conditions for $u$, so we conclude that $\psi=0$ or $\bar{u}^{1}=\tilde{u}$.

Let $\underline{S}^{1}$ satisfy

$$
\begin{aligned}
S_{t}-L^{S} S+m S & =m \hat{S}-\gamma^{-1} \tilde{u} f_{u}(\hat{S})=0 \\
d_{r}^{S} S_{r}+m S & =m \hat{S}-\gamma^{-1} \tilde{w} f_{w}(\hat{S})=0
\end{aligned}
$$

together with the other initial and boundary conditions for $S$; let $\underline{u}^{1}$ satisfy

$$
\begin{aligned}
u_{t} & =L^{u} u+u\left[f_{u}(\hat{S})-k\right], \\
d_{r}^{u} u_{r}+\alpha u & =H_{1}(\hat{S}, \hat{u}, \hat{w})=0,
\end{aligned}
$$

together with the other initial and boundary conditions for $u$; and let $\underline{w}^{1}$ satisfy

$$
w_{t}+\left(k_{w}+\beta\right) w=(1-W) H_{2}(\hat{S}, \hat{u}, \hat{w})=0,
$$

together with initial data for $w$.

Since $H_{2}(\hat{S}, \hat{u}, \hat{w}) \leq H_{2}(\tilde{S}, \tilde{u}, \tilde{w})$, the monotonicity lemma implies $0=\hat{w} \leq \underline{w}^{1} \leq$ $\bar{w}^{1}$. The positivity lemma implies $\underline{v}^{1} \geq 0$ for $v=S, u$.

$\phi=\bar{S}^{1}-\underline{S}^{1}$ satisfies

$$
\begin{aligned}
\phi_{t}-L^{S} \phi+m \phi & =m \tilde{S} \geq 0, \\
d_{r}^{S} \phi+m \phi & =m \tilde{S} \geq 0,
\end{aligned}
$$

together with homogeneous initial and boundary conditions for $S$, so we conclude that $\phi \geq 0$ and $\underline{S} \leq \bar{S}^{1}$.

$\psi=\bar{u}^{1}-\underline{u}^{1}$ satisfies

$$
\begin{aligned}
\psi_{t}-L^{u} \psi-\left[f_{u}(\tilde{S})-k\right] \psi & =\underline{u}^{1}\left[f_{u}(\tilde{S})-f_{u}(\hat{S})\right] \geq 0, \\
d_{r}^{u} \psi+\alpha \psi & =H_{1}(\tilde{Y})-H_{1}(\hat{Y}) \geq 0,
\end{aligned}
$$

together with homogeneous initial and boundary conditions for $u$, so we conclude that $\psi \geq 0$ from the positivity lemma or $\bar{u}^{1} \geq \underline{u}^{1}$.

Thus we have constructed $\underline{v}^{1}, \bar{v}^{1}$ for $v=S, u, w$ satisfying

$$
\hat{v} \leq \underline{v}^{1} \leq \bar{v}^{1} \leq \tilde{v} .
$$

Suppose that we have already constructed $\underline{v}^{j}, \bar{v}^{j}$ for $v=S, u, w$ and $j=1,2, \ldots, n$ satisfying

$$
\hat{v} \leq \underline{v}^{1} \leq \underline{v}^{2} \leq \cdots \leq \underline{v}^{n} \leq \bar{v}^{n} \leq \bar{v}^{n-1} \leq \cdots \leq \bar{v}^{1} \leq \tilde{v} .
$$

Let $\bar{S}^{n+1}$ satisfy

$$
\begin{aligned}
S_{t}-L^{S} S+m S & =m \bar{S}^{n}-\gamma^{-1} \underline{u}^{n} f_{u}\left(\bar{S}^{n}\right) \geq 0, \\
d_{r}^{S} S_{r}+m S & =m \bar{S}^{n}-\gamma^{-1} \underline{w}^{n} f_{w}\left(\bar{S}^{n}\right) \geq 0,
\end{aligned}
$$

together with the other initial and boundary conditions for $S$. The inequalities follow from (9.8) above. Let $\bar{u}^{n+1}$ satisfy

$$
\begin{aligned}
u_{t} & =L^{u} u+u\left[f_{u}\left(\bar{S}^{n}\right)-k\right], \\
d_{r}^{u} u_{r}+\alpha u & =H_{1}\left(\bar{Y}^{n}\right),
\end{aligned}
$$


together with the other initial and boundary conditions for $u$, and let $\bar{w}^{n+1}$ satisfy

$$
w_{t}+\left(k_{w}+\beta\right) w=(1-W) H_{2}\left(\bar{Y}^{n}\right),
$$

together with initial data for $w$.

Let $\underline{S}^{n+1}$ satisfy

$$
\begin{aligned}
S_{t}-L^{S} S+m S & =G_{n} \equiv m \underline{S}^{n}-\gamma^{-1} \bar{u}^{n} f_{u}\left(\underline{S}^{n}\right) \geq 0, \\
d_{r}^{S} S_{r}+m S & =F_{n} \equiv m \underline{S}^{n}-\gamma^{-1} \bar{w}^{n} f_{w}\left(\underline{S}^{n}\right) \geq 0,
\end{aligned}
$$

together with the other initial and boundary conditions for $S$. The inequalities follow from (9.8) above. Let $\underline{u}^{n+1}$ satisfy

$$
\begin{aligned}
u_{t} & =L^{u} u+u\left[f_{u}\left(\underline{S}^{n}\right)-k\right], \\
d_{r}^{u} u_{r}+\alpha u & =H_{1}\left(\underline{Y}^{n}\right),
\end{aligned}
$$

together with the other initial and boundary conditions for $u$, and let $\underline{w}^{n+1}$ satisfy

$$
w_{t}+\left(k_{w}+\beta\right) w=(1-W) H_{2}\left(\underline{Y}^{n}\right),
$$

together with initial data for $w$.

We must show that for $v=S, u, w$

$$
\underline{v}^{n} \leq \underline{v}^{n+1} \leq \bar{v}^{n+1} \leq \bar{v}^{n}, n \geq 1 .
$$

We note that $\underline{v}^{n+1} \geq 0, \bar{v}^{n+1} \geq 0$ for $v=S, u$ from the positivity lemma.

$\phi=\bar{S}^{n}-\bar{S}^{n+1}$ satisfies

$$
\begin{array}{rl}
\phi_{t}-L^{S} \phi+m \phi= & {\left[m \bar{S}^{n-1}-\gamma^{-1} \underline{u}^{n-1} f_{u}\left(\bar{S}^{n-1}\right)\right]-\left[m \bar{S}^{n}-\gamma^{-1} \underline{u}^{n} f_{u}\left(\bar{S}^{n}\right)\right]} \\
= & {\left[m \bar{S}^{n-1}-\gamma^{-1} \underline{u}^{n-1} f_{u}\left(\bar{S}^{n-1}\right)\right]} \\
& \quad-\left[m \bar{S}^{n}-\gamma^{-1} \underline{u}^{n-1} f_{u}\left(\bar{S}^{n}\right)\right]+\gamma^{-1} f_{u}\left(\bar{S}^{n}\right)\left(\underline{u}^{n}-\underline{u}^{n-1}\right) \\
\geq & 0, \\
d_{r}^{S} \phi+m \phi= & {\left[m \bar{S}^{n-1}-\gamma^{-1} \underline{w}^{n-1} f_{u}\left(\bar{S}^{n-1}\right)\right]-\left[m \bar{S}^{n}-\gamma^{-1} \underline{w}^{n} f_{u}\left(\bar{S}^{n}\right)\right]} \\
= & {\left[m \bar{S}^{n-1}-\gamma^{-1} \underline{w}^{n-1} f_{u}\left(\bar{S}^{n-1}\right)\right]} \\
& \quad-\left[m \bar{S}^{n}-\gamma^{-1} \underline{w}^{n-1} f_{u}\left(\bar{S}^{n}\right)\right]+\gamma^{-1} f_{u}\left(\bar{S}^{n}\right)\left(\underline{w}^{n}-\underline{w}^{n-1}\right) \\
\geq 0 & 0,
\end{array}
$$

together with homogeneous initial and boundary conditions for $S$, so we conclude that $\phi \geq 0$ and hence $\bar{S}^{n+1} \leq \bar{S}^{n}$. Here, we have used (9.8).

$\phi=\bar{S}^{n+1}-\underline{S}^{n+1}$ satisfies

$$
\begin{aligned}
\phi_{t}-L^{S} \phi+m \phi= & {\left[m \bar{S}^{n}-\gamma^{-1} \underline{u}^{n} f_{u}\left(\bar{S}^{n}\right)\right]-\left[m \underline{S}^{n}-\gamma^{-1} \bar{u}^{n} f_{u}\left(\underline{S}^{n}\right)\right] } \\
= & {\left[m \bar{S}^{n}-\gamma^{-1} \underline{u}^{n} f_{u}\left(\bar{S}^{n}\right)\right] } \\
& \quad-\left[m \underline{S}^{n}-\gamma^{-1} \underline{u}^{n} f_{u}\left(\underline{S}^{n}\right)\right]+\gamma^{-1} f_{u}\left(\underline{S}^{n}\right)\left(\bar{u}^{n}-\underline{u}^{n}\right) \\
\geq & \left.0, \quad \bar{S}^{n}-\gamma^{-1} \underline{w}^{n} f_{w}\left(\bar{S}^{n}\right)\right]-\left[m \underline{S}^{n}-\gamma^{-1} \bar{w}^{n} f_{w}\left(\underline{S}^{n}\right)\right] \\
d_{r}^{S} \phi+m \phi= & {\left[m \bar{S}^{n}-\gamma^{-1} \underline{w}^{n} f_{w}\left(\bar{S}^{n}\right)\right] } \\
& \quad-\left[m \underline{S}^{n}-\gamma^{-1} \underline{w}^{n} f_{w}\left(\underline{S}^{n}\right)\right]+\gamma^{-1} f_{w}\left(\underline{S}^{n}\right)\left(\bar{w}^{n}-\underline{w}^{n}\right) \\
\geq & 0,
\end{aligned}
$$


together with homogeneous initial and boundary conditions for $S$, so we conclude that $\phi \geq 0$ and hence $\bar{S}^{n+1} \geq \underline{S}^{n+1}$. Here, again, we have used (9.8).

$\phi=\underline{S}^{n+1}-\underline{S}^{n}$ satisfies

$$
\begin{aligned}
\phi_{t}-L^{S} \phi+m \phi= & {\left[m \underline{S}^{n}-\gamma^{-1} \bar{u}^{n} f_{u}\left(\underline{S}^{n}\right)\right]-\left[m \underline{S}^{n-1}-\gamma^{-1} \bar{u}^{n-1} f_{u}\left(\underline{S}^{n-1}\right)\right] } \\
= & {\left[m \underline{S}^{n}-\gamma^{-1} \bar{u}^{n} f_{u}\left(\underline{S}^{n}\right)\right] } \\
& \quad-\left[m \underline{S}^{n-1}-\gamma^{-1} \bar{u}^{n} f_{u}\left(\underline{S}^{n-1}\right)\right]+\gamma^{-1} f_{u}\left(\underline{S}^{n-1}\right)\left(\bar{u}^{n-1}-\bar{u}^{n}\right) \\
\geq & 0, \quad \\
d_{r}^{S} \phi+m \phi= & {\left[m \underline{S}^{n}-\gamma^{-1} \bar{w}^{n} f_{w}\left(\underline{S}^{n}\right)\right]-\left[m \underline{S}^{n-1}-\gamma^{-1} \bar{w}^{n-1} f_{w}\left(\underline{S}^{n-1}\right)\right] } \\
= & {\left[m \underline{S}^{n}-\gamma^{-1} \bar{w}^{n} f_{w}\left(\underline{S}^{n}\right)\right] } \\
& \quad-\left[m \underline{S}^{n-1}-\gamma^{-1} \bar{w}^{n} f_{w}\left(\underline{S}^{n-1}\right)\right]+\gamma^{-1} f_{w}\left(\underline{S}^{n-1}\right)\left(\bar{w}^{n-1}-\bar{w}^{n}\right) \\
\geq & 0,
\end{aligned}
$$

together with homogeneous initial and boundary conditions for $S$, so we conclude that $\phi \geq 0$ and hence $\underline{S}^{n+1} \geq \underline{S}^{n}$.

$\psi=\bar{u}^{n}-\bar{u}^{n+1}$ satisfies

$$
\begin{aligned}
\psi_{t}-L^{u} \psi & =\left[f_{u}\left(\bar{S}^{n-1}\right)-k\right] \bar{u}^{n}-\left[f_{u}\left(\bar{S}^{n}\right)-k\right] \bar{u}^{n+1} \\
& =\left[f_{u}\left(\bar{S}^{n-1}\right)-k\right] \psi+\left[f_{u}\left(\bar{S}^{n-1}\right)-f_{u}\left(\bar{S}^{n}\right)\right] \bar{u}^{n+1} \\
& \geq\left[f_{u}\left(\bar{S}^{n-1}\right)-k\right] \psi, \\
d_{r}^{u} \psi+\alpha \psi & =H_{1}\left(\bar{Y}^{n-1}\right)-H_{1}\left(\bar{Y}^{n}\right) \geq 0,
\end{aligned}
$$

together with homogeneous initial and boundary conditions for $u$, so we conclude that $\psi \geq 0$ from the positivity lemma or $\bar{u}^{n} \geq \bar{u}^{n+1}$.

$\psi=\bar{u}^{n+1}-\underline{u}^{n+1}$ satisfies

$$
\begin{aligned}
\psi_{t}-L^{u} \psi & =\left[f_{u}\left(\bar{S}^{n}\right)-k\right] \bar{u}^{n+1}-\left[f_{u}\left(\underline{S}^{n}\right)-k\right] \underline{u}^{n+1} \\
& =\left[f_{u}\left(\bar{S}^{n}\right)-k\right] \psi+\left[f_{u}\left(\bar{S}^{n}\right)-f_{u}\left(\underline{S}^{n}\right)\right] \underline{u}^{n+1} \\
& \geq\left[f_{u}\left(\bar{S}^{n}\right)-k\right] \psi, \\
d_{r}^{u} \psi+\alpha \psi & =H_{1}\left(\bar{Y}^{n}\right)-H_{1}\left(\underline{Y}^{n}\right) \geq 0,
\end{aligned}
$$

together with homogeneous initial and boundary conditions for $u$, so we conclude that $\psi \geq 0$ from the positivity lemma or $\bar{u}^{n+1} \geq \underline{u}^{n+1}$.

$\psi=\underline{u}^{n+1}-\underline{u}^{n}$ satisfies

$$
\begin{aligned}
\psi_{t}-L^{u} \psi & =\left[f_{u}\left(\underline{S}^{n}\right)-k\right] \underline{u}^{n+1}-\left[f_{u}\left(\underline{S}^{n-1}\right)-k\right] \underline{u}^{n} \\
& =\left[f_{u}\left(\underline{S}^{n}\right)-k\right] \psi+\left[f_{u}\left(\underline{S}^{n}\right)-f_{u}\left(\underline{S}^{n-1}\right)\right] \underline{u}^{n} \\
& \geq\left[f_{u}\left(\underline{S}^{n}\right)-k\right] \psi, \\
d_{r}^{u} \psi+\alpha \psi & =H_{1}\left(\underline{Y}^{n}\right)-H_{1}\left(\underline{Y}^{n-1}\right) \geq 0,
\end{aligned}
$$

together with homogeneous initial and boundary conditions for $u$, so we conclude that $\psi \geq 0$ from the positivity lemma or $\underline{u}^{n+1} \geq \underline{u}^{n}$.

Finally, since

$$
H_{2}\left(\underline{Y}^{n-1}\right) \leq H_{2}\left(\underline{Y}^{n}\right) \leq H_{2}\left(\bar{Y}^{n}\right) \leq H_{2}\left(\bar{Y}^{n-1}\right),
$$

the monotonicity lemma implies that

$$
\underline{w}^{n} \leq \underline{w}^{n+1} \leq \bar{w}^{n+1} \leq \bar{w}^{n} .
$$


We have established the existence of monotone sequences $\left\{\bar{v}^{n}\right\},\left\{\underline{v}^{n}\right\}$ for $v=$ $S, u, w$ and, evidently,

$$
\bar{v}^{n} \searrow \bar{v}, \quad \underline{v}^{n} \nearrow \underline{v}
$$

in a pointwise sense and

$$
\hat{v} \leq \underline{v}^{n} \leq \underline{v} \leq \bar{v} \leq \bar{v}^{n} \leq \tilde{v}, \quad n \geq 1 .
$$

In fact, by Lemma 9.1 and the uniform boundedness of the sequence, the sequences $\left\{\bar{v}^{n}\right\},\left\{\underline{v}^{n}\right\}$ for $v=S, u$ have bounded Hölder norms in $\bar{\Omega} \times\left[T^{\prime}, T\right]$ for each $0<T^{\prime}<T$ and therefore converge uniformly on each such set. As a consequence, the same holds for the upper and lower sequence of $w$ 's.

Consider, for example, equation (9.11) for the sequence $\underline{S}^{n+1}$. As the left-hand side of (9.11) is uniformly bounded on $D_{T}$, one can argue (see, e.g., (9.18) below) that

$$
\left\|\underline{S}^{n}\right\|_{V_{2}\left(D_{T}\right)} \leq C
$$

for all $n$. A similar argument applies to the sequences $\bar{S}^{n}, \underline{u}^{n}, \bar{u}^{n}$; they are uniformly bounded in $V_{2}$. From this we may conclude the weak convergence of some (common) subsequence of $\underline{S}^{n}, \bar{S}^{n}, \underline{u}^{n}, \bar{u}^{n}$. By passing to the limit along this subsequence in the weak formulation of the $S$ and $u$ equations (see below Lemma 9.1) we find that this subsequence converges weakly in $V_{2}$ (and uniformly on $\bar{\Omega} \times\left[T^{\prime}, T\right]$ ) to a weak solution $(\underline{Y}, \bar{Y})$ of the system

$$
\begin{aligned}
\underline{S}_{t} & =L^{S} \underline{S}-\gamma^{-1} \bar{u} f_{u}(\underline{S}), \\
\underline{u}_{t} & =L^{u} \underline{u}+\underline{u}\left[f_{u}(\underline{S})-k\right], \\
\underline{w}_{t}+\left(k_{w}+\beta\right) \underline{w} & =(1-\underline{W}) H_{2}(\underline{Y}), \\
\bar{S}_{t} & =L^{S} \bar{S}-\gamma^{-1} \underline{u} f_{u}(\bar{S}), \\
\bar{u}_{t} & =L^{u} \bar{u}+\bar{u}\left[f_{u}(\bar{S})-k\right], \\
\bar{w}_{t}+\left(k_{w}+\beta\right) \bar{w} & =(1-\bar{W}) H_{2}(\bar{Y})
\end{aligned}
$$

with the radial boundary conditions

$$
\begin{aligned}
d_{r}^{S} \underline{S}_{r} & =-\gamma^{-1} \bar{w} f_{w}(\underline{S}), \\
d_{r}^{u} \underline{u}_{r}+\alpha \underline{u} & =H_{1}(\underline{Y}), \\
d_{r}^{S} \bar{S}_{r} & =-\gamma^{-1} \underline{w} f_{w}(\bar{S}), \\
d_{r}^{u} \bar{u}_{r}+\alpha \bar{u} & =H_{1}(\bar{Y}),
\end{aligned}
$$

Danckwerts' boundary conditions at $x=0, L$, as well as initial conditions

$$
\begin{aligned}
& \underline{S}=\bar{S}=S_{0}, \\
& \underline{u}=\bar{u}=u_{0}, t=0, \\
& \underline{w}=\bar{w}=w_{0} .
\end{aligned}
$$

Now, consider the system

$$
\begin{aligned}
S_{t}^{1} & =L^{S} S^{1}-\gamma^{-1} u^{2} f_{u}\left(S^{1}\right), \\
u_{t}^{1} & =L^{u} u^{1}+u^{1}\left[f_{u}\left(S^{1}\right)-k\right], \\
w_{t}^{1}+\left(k_{w}+\beta\right) w^{1} & =\left(1-W^{1}\right) H_{2}\left(Y^{1}\right), \\
S_{t}^{2} & =L^{S} S^{2}-\gamma^{-1} u^{1} f_{u}\left(S^{2}\right), \\
u_{t}^{2} & =L^{u} u^{2}+u^{2}\left[f_{u}\left(S^{2}\right)-k\right], \\
w_{t}^{2}+\left(k_{w}+\beta\right) w^{2} & =\left(1-W^{2}\right) H_{2}\left(Y^{2}\right)
\end{aligned}
$$


with the radial boundary conditions

$$
\begin{aligned}
d_{r}^{S} S_{r}^{1} & =-\gamma^{-1} w^{2} f_{w}\left(S^{1}\right), \\
d_{r}^{u} u_{r}^{1}+\alpha u^{1} & =H_{1}\left(Y^{1}\right), \\
d_{r}^{S} S_{r}^{2} & =-\gamma^{-1} w^{1} f_{w}\left(S^{2}\right), \\
d_{r}^{u} u_{r}^{2}+\alpha u^{2} & =H_{1}\left(Y^{2}\right),
\end{aligned}
$$

the usual boundary conditions at $x=0, L$, as well as the initial conditions

$$
\begin{aligned}
S^{1} & =S^{2}=S_{0}, \\
u^{1} & =u^{2}=u_{0}, t=0, \\
w^{1} & =w^{2}=w_{0} .
\end{aligned}
$$

If $\left(Y^{1}, Y^{2}\right)$ satisfies (9.13)-(9.15), then by symmetry, so does $\left(Y^{2}, Y^{1}\right)$. Therefore, if (9.13)-(9.15) has a unique solution, then $Y^{1}=Y^{2}$. But $\left(Y^{1}, Y^{2}\right)=(\underline{Y}, \bar{Y})$ satisfies (9.13)-(9.15). Thus,

$$
\underline{Y}=\bar{Y}
$$

so we have a solution $Y=\underline{Y}=\bar{Y}$.

We need a uniqueness result for (9.13)-(9.15). To simplify the presentation, we consider the model system

$$
\begin{aligned}
u_{t} & =L u+f(x, t, u), \quad(x, t) \in D_{T}, \\
u_{r} & =g(x, t, u, w), \quad(x, t) \in S_{T}=\partial_{r} \Omega \times(0, T), \\
w_{t} & =h(x, t, u, w), \quad(x, t) \in S_{T}, \\
u(x, 0) & =u_{0}(x), \quad w(x, 0)=w_{0}(x),
\end{aligned}
$$

where, for simplicity of notation, we let $x$ represent the point $(x, y, z)$. Homogeneous boundary conditions (2.4) and (2.5) are assumed to hold. Entirely similar arguments apply to (9.13)-(9.15). We assume that $f, g, h$ are Lipschitz functions in $u, w$ with Lipschitz constant $K$ (notice that $u, S, w$ are bounded on $D_{T}$ ). To prove the uniqueness we can assume that $u_{0}, w_{0}$ are identically zero and show that, for small $T, u, w$ are zero functions. First, we consider the parabolic equation

$$
\begin{aligned}
& u_{t}=L u+F(x, t), \quad(x, t) \in D_{T}, \\
& u_{r}=G(x, t), \quad(x, t) \in S_{T} .
\end{aligned}
$$

Using the Steklov average and taking to the limit (see [22], pp. 141-142), we can test the weak formulation of this equation (see below Lemma 9.1) by $\eta=u$. By a similar argument to that of Ladyzenskaya's Lemma 2.1 of Chapter 3, using the facts

$$
\int_{S_{T}} G u d A d t \leq\|G\|_{2, S_{T}}\|u\|_{2, S_{T}}, \quad \int_{S_{T}} u^{2} d A d t \leq C\|u\|_{V_{2}\left(D_{T}\right)}^{2},
$$

one can show that any $V_{2}$ solution of (9.17) satisfies

$$
\|u\|_{V_{2}\left(D_{T}\right)} \leq C\left[\|u(x, 0)\|_{2, \Omega}+\|F\|_{2, D_{T}}+\|G\|_{2, S_{T}}\right] .
$$

Applying this to the equation for $u$ in $(9.16)$, with $u(x, 0) \equiv 0$, we obtain

$$
\|u\|_{V_{2}\left(D_{T}\right)}^{2} \leq C\left[\|f\|_{2, D_{T}}^{2}+\|g\|_{2, S_{T}}^{2}\right] .
$$


By the Lipschitz condition, we have

$$
\|f\|_{2, D_{T}}^{2} \leq K^{2}\|u\|_{2, D_{T}}^{2} \leq K^{2} T \max _{t \in(0, T)}\|u(\cdot, t)\|_{\Omega}^{2}
$$

and

$$
\begin{aligned}
\|g\|_{2, S_{T}}^{2} & \leq K^{2}\left(\|u\|_{2, S_{T}}^{2}+\|w\|_{2, S_{T}}^{2}\right) \\
& \leq K^{2}\left(\|u\|_{2, S_{T}}^{2}+T \max _{t \in(0, T)} \int_{\partial_{r} \Omega} w^{2}(x, t) d A\right) .
\end{aligned}
$$

Since $w(x, 0) \equiv 0$, we have

$$
w(x, t)=\int_{0}^{t} h(x, s, u(x, s), w(x, s)) d s \quad \text { for all } t \in(0, T) .
$$

Integrating over $\partial_{r} \Omega$ and using the Hölder inequality and the Lipschitz condition, we have

$$
\int_{\partial_{r} \Omega} w^{2}(x, t) d A \leq \int_{\partial_{r} \Omega} t \int_{0}^{t} h^{2}(x, s, u, w) d A d s \leq K^{2} T\left[\|u\|_{2, S_{T}}^{2}+\|w\|_{2, S_{T}}^{2}\right] .
$$

This implies that

$$
\max _{t \in(0, T)} \int_{\partial_{r} \Omega} w^{2}(x, t) d A \leq K^{2} T\left[\|u\|_{2, S_{T}}^{2}+T \max _{t \in(0, T)} \int_{\partial_{r} \Omega} w^{2}(x, t) d A\right] .
$$

Thus, if $T$ is small, we obtain

$$
\max _{t \in(0, T)} \int_{\partial_{r} \Omega} w^{2}(x, t) d A \leq C\|u\|_{2, S_{T}}^{2} .
$$

By (9.21) and the above, we get

$$
\|g\|_{2, S_{T}}^{2} \leq C K^{2}(1+T)\|u\|_{2, S_{T}}^{2} .
$$

Altogether, the above and (9.19) and (9.20) imply

$$
\|u\|_{V_{2}\left(D_{T}\right)}^{2} \leq C\left[K^{2} T \max _{t \in(0, T)}\|u\|_{2, \Omega}^{2}+C K^{2}(1+T)\|u\|_{2, S_{T}}^{2}\right] .
$$

We now use the inequality

$$
\|u\|_{2, S_{T}}^{2} \leq \epsilon\|u\|_{V_{2}\left(D_{T}\right)}^{2}+C(\epsilon)\|u\|_{2, D_{T}}^{2}
$$

with sufficiently small $\epsilon$ to derive from (9.23) that

$$
\|u\|_{V_{2}\left(D_{T}\right)}^{2} \leq C_{1}\left[K^{2} T \max _{t \in(0, T)}\|u\|_{2, \Omega}^{2}+\|u\|_{2, D_{T}}^{2}\right] \leq C_{2} T \max _{t \in(0, T)}\|u\|_{2, \Omega}^{2} .
$$

Since $\|u\|_{V_{2}\left(D_{T}\right)}^{2}=\max _{t \in(0, T)}\|u\|_{2, \Omega}^{2}+\|D u\|_{2, D_{T}}^{2}$, the above gives

$$
\max _{t \in(0, T)}\|u\|_{2, \Omega}^{2} \leq C_{2} T \max _{t \in(0, T)}\|u\|_{2, \Omega}^{2} .
$$

Thus, if $T$ is small, we must have $u \equiv 0$. The proof of uniqueness is completed. 
Proof of Theorem 3.6. In the construction of the upper solution $\tilde{S}$ above we observed that as $\tilde{S}$ satisfies

$$
\begin{aligned}
S_{t} & =L^{S} S, \\
0 & =d_{r}^{S} S_{r},
\end{aligned}
$$

together with (2.4), (2.5), and $S \geq 0$ at $t=0$, it follows that $S \rightarrow S^{0}$ as $t \rightarrow \infty$ uniformly in $\bar{\Omega}$. This follows from a simple comparison argument using the positivity lemma and the fact that the associated elliptic operator with homogeneous Danckwerts' boundary conditions has a negative principal eigenvalue. By continuity, the principal eigenvalue of the elliptic operator associated with the parabolic problem

$$
\begin{aligned}
S_{t} & =L^{S} S-m S, \\
0 & =d_{r}^{S} S_{r}+m S
\end{aligned}
$$

is also negative when $m>0$ is sufficiently small. As a consequence, a solution of this parabolic problem satisfying the usual boundary conditions at $x=0$ and $x=L$ and $S \geq 0$ when $t=0$ converges uniformly in $\Omega$ to the unique steady state $S_{m}$ of the associated elliptic problem. Moreover, $\phi=S_{m}-S^{0}$ satisfies

$$
\begin{aligned}
-L^{S} \phi+m \phi & =-m S^{0}, \\
d_{r}^{S} \phi_{r}+m \phi & =-m S^{0},
\end{aligned}
$$

and homogeneous Danckwerts' boundary conditions. Thus we conclude that $\phi=$ $O\left(m S^{0}\right)$, so $S_{m}=S^{0}[1-O(m)]$ for small $m>0$, where $m^{-1} O(m)$ is bounded for small $m>0$.

By (3.13), we can choose $\eta>0$ and $\mu>0$ so small that

$$
f_{u}\left(S^{0}-2 \eta\right)-k-\lambda_{u}>\mu \text { and } f_{w}\left(S^{0}-2 \eta\right)-k_{w}>\mu .
$$

Let $m>0$ be such that $\left\|S_{m}-S^{0}\right\|_{\infty}<\eta$ and let $\delta>0$ be so small that

$$
m S-\gamma^{-1} \delta f(S) \geq 0, \quad S \geq 0
$$

for $f=f_{u}$ and $f=f_{w}$.

If our assertion is false, then there is a nontrivial solution $(S, u, w)$ and $t_{0} \geq 0$ such that $u, w \leq \delta$, uniformly on their respective domains, for $t \geq t_{0}$. By our choice of $\delta$ and (9.25), it follows that, for $t \geq t_{0}, S$ satisfies

$$
\begin{aligned}
S_{t}-L^{S} S+m S & \geq 0, \\
d_{r}^{S} S_{r}+m S & \geq 0,
\end{aligned}
$$

together with (2.4), (2.5) and $S \geq 0$ at $t=t_{0}$. On comparing with the solution $S=S^{*}$ of the associated differential equality ( $S^{*}$ converges uniformly to $S_{m}$ as noted above) using the positivity lemma, we find that $S \geq S^{*}$ for $t \geq t_{0}$ and $S^{*} \geq S_{m}-\eta$ for $t \geq t_{1}$ for some $t_{1} \geq t_{0}$. Therefore, $S \geq S^{0}-2 \eta$ for $t \geq t_{1}$. We will obtain a contradiction to this estimate by making use of the arguments leading to Theorem 3.1.

Using the notation of Theorem 3.1, observe that for $t \geq t_{1}$ we have

$$
\left.(Y+Z)^{\prime}=-\lambda^{u} Y+\int_{\Omega} u \psi\left[f_{u}(S)-k\right] d V-\left(k_{w}+\beta\right) Z+\int_{\partial_{r} \Omega} \psi w\left[f_{w}(S)+\beta\right]\right) d A
$$




$$
\begin{aligned}
& \geq-\lambda^{u} Y+\int_{\Omega} u \psi\left[f_{u}\left(S^{0}-2 \eta\right)-k\right] d V-\left(k_{w}+\beta\right) Z \\
& \quad+\int_{\partial_{r} \Omega} \psi w\left[f_{w}\left(S^{0}-2 \eta\right)+\beta\right] d A \\
& =\left[f_{u}\left(S^{0}-2 \eta\right)-k-\lambda_{u}\right] Y+\left[f_{w}\left(S^{0}-2 \eta\right)-k_{w}\right] Z \\
& \geq \mu(Y+Z),
\end{aligned}
$$

where we have used that $\lambda_{u}=\lambda^{u}$. Consequently, $Y+Z$ diverges to positive infinity, contradicting $u, w \leq \delta$ for $t \geq t_{0}$.

Acknowledgments. The authors would like to acknowledge useful conversations with C. Cosner and G. Lieberman.

\section{REFERENCES}

[1] H. Amann, Fixed point equations and nonlinear eigenvalue problems in ordered Banach spaces, SIAM Rev. 18 (1976), pp. 620-709.

[2] H. Amann, Nonlinear elliptic equations with nonlinear boundary conditions, in New Developments in Differential Equations, W. Eckhaus, ed., North-Holland, 1976, pp. 43-63.

[3] R. ARIs, Mathematical Modeling, a Chemical Engineer's Perspective, Academic Press, New York, 1999.

[4] M. Ballyk And H. L. Smith, A Flow Reactor with Wall Growth, in Mathematical Models in Medical and Health Science, M. Horn, G. Simonett, and G. Webb, eds., Vanderbilt University Press, Nashville, TN, 1998, pp. 17-28.

[5] M. Ballyk And H. L. Smith, A model of microbial growth in a plug flow reactor with wall attachment, Mathematical Biosciences, 158 (1999), pp. 95-126.

[6] M. Ballyk, D. Le, D. A. Jones, AND H. L. SMith, Effects of random motility on microbial growth and competition in a flow reactor, SIAM J. Appl. Math., 59 (1998), pp. 573-596.

[7] M. Ballyk, D. Jones, AND H. L. SMith, Microbial competition in reactors with wall attachment: A comparison of chemostat and plug flow models, Microbial Ecology, 41 (2001), pp. 210-221.

[8] B. Baltzis And A. Fredrickson, Competition of two microbial populations for a single resource in a chemostat when one of them exhibits wall attachment, Biotechnology and Bioengineering, XXV (1983), pp. 2419-2439.

[9] H. Berg, Random Walks in Biology, Princeton University Press, Princeton, NJ, 1983.

[10] J. Bryers, ED., Biofilms II: Process Analysis and Applications, Wiley Series in Ecol. and Appl. Microbiology, Wiley, New York, 2000.

[11] J. Costerton, P. Stewart, and E. Greenberg, Bacterial biofilms: A common cause of persistent infections, Science, 284 (1999), pp. 1318-1322.

[12] J. Costerton, Overview of microbial biofilms, J. Indust. Microbiol., 15 (1995), pp. 137-140.

[13] R. Dillon, L. Fauci, A. Fogelson, And D. Gaver, Modeling biofilm processes using the immersed boundary method, J. Comput. Phys., 129 (1996), pp. 57-73.

[14] J. Dockery AND I. Klapper, Finger formation in biofilm layers, SIAM J. Appl. Math., 62 (2001), pp. 853-869.

[15] Dung Le And H. L. Smith, Strong positivity property of solutions to parabolic and elliptic equations on nonsmooth domains, J. Math. Anal. Appl., to appear.

[16] Dung Le, Remarks on Holder continuity for parabolic equations and convergence to global attractors, Nonlinear Anal., 41 (2000), pp. 921-941.

[17] L. Fraenkel, Introduction to Maximum Principles and Symmetry in Elliptic Problems, Cambridge University Press, Cambridge, UK, 2000.

[18] R. FreTER, Mechanisms that control the microflora in the large intestine, in Human Intestinal Microflora in Health and Disease, D. Hentges, ed., Academic Press, New York, 1983, pp. $33-54$.

[19] R. Freter, H. Brickner, And S. Temme, An understanding of colonization resistance of the mammalian large intestine requires mathematical analysis, Microecology and Therapy, 16 (1986), pp. $147-155$.

[20] D. Gilbarg and L. Hormander, Intermediate Schauder estimates, Arch. Ration. Mech. Anal., 74 (1980), pp. 297-318. 
[21] D. Gilbarg and N. Trudinger, Elliptic Partial Differential Equations of Second Order, Springer-Verlag, New York, 1977.

[22] O. Ladyzhenskaya and N. Ural'tseva, Linear and Quasilinear Elliptic Equations, Academic Press, New York, 1968.

[23] M. Krasnoselski, Positive Solutions of Operator Equations, P. Noordhoff, Groningen, The Netherlands, 1964.

[24] G. Lieberman, Oblique derivative problems in Lipschitz domains II. Discontinuous boundary data, J. Reine Angew. Math., 389 (1988), pp. 1-21.

[25] R. Nussbaum, Positive operators and elliptic eigenvalue problems, Math. Z., 186 (1984), pp. 247-264.

[26] R. Nussbaum, A periodicity threshold theorem for some nonlinear integral equations, SIAM J. Math. Anal., 9 (1978), pp. 356-376.

[27] C. PAO, Nonlinear Parabolic and Elliptic Equations, Plenum Press, New York, 1992.

[28] M. Protter and H. Weinberger, Maximum Principles in Differential Equations, Prentice Hall, Englewood Cliffs, NJ, 1967.

[29] D. A. Jones And H. L. Smith, Microbial competition for nutrient and wall sites in plug flow, SIAM J. Appl. Math., 60 (2000), pp. 1576-1600.

[30] E. D. Stemmons And H. L. Smith, Competition in a chemostat with wall attachment, SIAM J. Appl. Math., 61 (2000), pp. 567-595.

[31] P. Stewart, G. MCFeters, and C.-T. Huang, Biofilm control by antimicrobial agents, in Biofilms II: Process Analysis and Applications, J. Bryers, ed., Wiley, New York, 2000, Chapter 11.

[32] J. Gatica And H. L. Smith, Fixed point techniques in a cone with applications, J. Math. Anal. Appl., 61 (1977), pp. 58-71.

[33] Dung Le, H. L.Smith, and P. Waltman, Growth in the unstirred chemostat with different diffusion rates, Fields Inst. Commun., 21 (1999), pp. 131-142. 